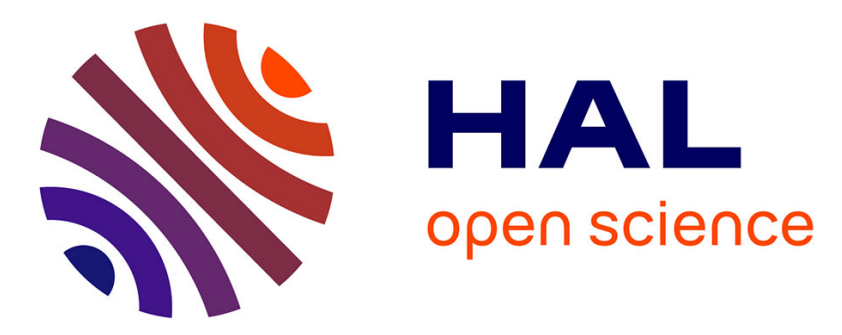

\title{
Magnetic stabilization of the buoyant convection between infinite horizontal walls with a horizontal temperature gradient
}

\author{
S. Kaddeche, Daniel Henry, H. Ben Hadid
}

\section{- To cite this version:}

S. Kaddeche, Daniel Henry, H. Ben Hadid. Magnetic stabilization of the buoyant convection between infinite horizontal walls with a horizontal temperature gradient. Journal of Fluid Mechanics, 2003, 480, pp.185-216. 10.1017/S0022112002003622 . hal-00140473

\section{HAL Id: hal-00140473 \\ https://hal.science/hal-00140473}

Submitted on 6 Apr 2007

HAL is a multi-disciplinary open access archive for the deposit and dissemination of scientific research documents, whether they are published or not. The documents may come from teaching and research institutions in France or abroad, or from public or private research centers.
L'archive ouverte pluridisciplinaire HAL, est destinée au dépôt et à la diffusion de documents scientifiques de niveau recherche, publiés ou non, émanant des établissements d'enseignement et de recherche français ou étrangers, des laboratoires publics ou privés. 


\title{
Magnetic stabilization of the buoyant convection between infinite horizontal walls with a horizontal temperature gradient
}

\author{
By S. KADDECHE ${ }^{1}$, D. HENRY ${ }^{2} \dagger$ AND H. BENHADID \\ ${ }^{1}$ Institut National des Sciences Appliquées et de Technologie, BP 676, 1080 Tunis Cedex, Tunisie \\ ${ }^{2}$ Laboratoire de Mécanique des Fluides et d'Acoustique, UMR-CNRS 5509, Ecole Centrale de \\ Lyon/Université Claude Bernard, Lyon 1, ECL-BP 163, 69131 Ecully Cedex, France
}

(Received 8 March 2002 and in revised form 17 October 2002)

Buoyant convection induced between infinite horizontal walls by a horizontal temperature gradient is characterized by simple monodimensional parallel flows. In a layer of low-Prandtl-number fluid, these flows can involve two types of instabilities: two-dimensional stationary transverse instabilities and three-dimensional oscillatory longitudinal instabilities. The stabilization of such flows by a constant magnetic field (vertical, or horizontal with a direction transverse or longitudinal to the flow) is investigated in this paper through a linear stability analysis and energy considerations. The vertical magnetic field stabilizes the instabilities more quickly than the horizontal fields, but the stabilization is only obtained up to moderate values of Hartmann number $H a$ (before disappearance of the instabilities). Characteristic laws, given by the critical Grashof number $\mathrm{Gr}_{c}$ as a function of $\mathrm{Ha}$ (proportional to the intensity of the magnetic field), have been found for the initial stabilization at small $\mathrm{Ha}$. They are $G r_{c} \sim G r_{c_{0}} \exp \left(H a^{2}\right)$ for the two-dimensional instabilities and $G r_{c}-G r_{c_{0}} \sim H a^{2}$ for the three-dimensional instabilities (where $G r_{c_{0}}$ is the critical Grashof number at $H a=0$ ), indicating that the three-dimensional instabilities, less stabilized, will prevail in a vertical magnetic field. It has been shown by an energy analysis that the strong stabilization of the two-dimensional instabilities is connected to the strong diminution of the destabilizing shear energy term when the velocity profiles are modified by the vertical magnetic field, and affected little by the Lorentz energy term. For the horizontal magnetic fields, the stabilization is very weak at small $H a$, but then reaches an asymptotic behaviour corresponding to $G r_{c} \sim H a$. This asymptotic stabilization is connected to the decrease of the destabilizing shear energy term due to the increase of the marginal cell length in the horizontal magnetic field. In fact, this stabilization only concerns the two-dimensional modes in the longitudinal field and the three-dimensional modes in the transverse field.

\section{Introduction}

The flow created by lateral heating in a cavity is one of the classical heat and mass transfer problems with significance for fundamental fluid mechanics as well as for geophysical and engineering applications. Among engineering applications there are materials processing, crystal growth, cooling systems for nuclear reactors, solar 
energy collectors, manufacturing and welding. An increased interest in this field came from the need to understand the hydrodynamical aspects of semiconductor crystal growth, particularly for the horizontal Bridgman technique. In the crystal growth process the existence of spontaneous oscillations of the flow has been demonstrated experimentally (see Pimputkar \& Ostrach 1981) and these oscillations are responsible for the occurrence of layered variations of impurities in crystals, known as striations. Similar flow oscillations have been identified experimentally in cavities filled with either mercury or gallium as working fluid (Hart 1983; Hung \& Andereck 1988; Hurle, Jakeman \& Johnson 1974).

The dynamical behaviour of such flows driven by horizontal temperature gradients in conducting fluids has attracted the attention of researchers for many years. In a long cavity and for a small temperature difference, convection is characterized by a parallel core flow (Cormack, Leal \& Imberger 1974) which is susceptible to a variety of instabilities above certain critical values of the Grashof number. First stability studies concerned the monodimensional parallel flow obtained in an infinite layer. The work was initiated by Hart (1972) who showed, using linear theory, that for rigid boundaries the instability sets in either as stationary transverse cells or as oscillatory longitudinal rolls, depending on the value of the Prandtl number. Transverse cells have axes perpendicular to the base flow direction and then a two-dimensional flow structure persists, whereas the axes of longitudinal rolls are aligned with the base flow which leads to a global three-dimensional flow structure. Further work on stability (see Pimputkar \& Ostrach 1981 for an extensive review) has been performed in this field. For example for a free-surface layer with top and bottom insulated boundaries, it has been shown that for Prandtl numbers $\operatorname{Pr}$ less than 0.0045 oscillatory transverse instabilities are the dominant mode, whereas the longitudinal oscillatory mode dominates in the range $0.0045 \leqslant P r \leqslant 0.41$. More precisely, for the longitudinal instability, travelling waves become the preferred mode for $0.0045 \leqslant P r \leqslant 0.38$, while standing waves prevail for $0.38 \leqslant P r \leqslant 0.41$ (Laure \& Roux 1987). Some theoretical studies have also been performed. The onset of the longitudinal rolls was studied by Gill (1974). He examined the stability characteristics in a qualitative way in the small Prandtl number limit and related his findings to the oscillatory flows observed by Hurle (1966) and Hurle et al. (1974). Note that the theory of Gill (1974) provides results that agree within a factor two with the numerical results found by Hart (1972) for the case of a layer between rigid boundaries.

Smith \& Davis (1983) have investigated the stability of thermocapillary flows generated by a horizontal temperature gradient imposed along a thin liquid layer of infinite horizontal extent bounded below by an adiabatic rigid surface. They reported on two classes of instabilities which differ by the energy transfer mechanism. The first mode is a thermal instability which takes the form of either longitudinal rolls or hydrothermal waves. The second mode is closely related to free-surface deformations and involves mechanical transfer of momentum. The analysis of hydrothermal wave instability was extended by Priede \& Gerbeth (1997b) to different types of thermal boundary conditions which are shown to have an important effect on the instability threshold. These authors then studied the influence of uniform magnetic fields, either coplanar to the liquid layer (i.e. horizontal) (Priede \& Gerbeth 1995, 1997a) or transverse (i.e. vertical) (Priede, Thess \& Gerbeth 1994; Priede \& Gerbeth 2000). They have shown that the vertical field is more effective in stabilizing hydrothermal waves (the threshold increasing asymptotically as square of the field strength), whereas the horizontal field, which stabilizes all disturbances except those aligned with the field, leads to the alignment of the most unstable disturbance along the magnetic flux lines. 
The stability of buoyant-thermocapillary-driven flows has been considered by Laure \& Roux (1989) for low-Prandtl-number fluids, then by Gershuni et al. (1992) and by Parmentier, Régnier \& Lebon (1993) for larger values of the Prandtl number. The characteristics and the stability of two-dimensional buoyant-thermocapillary-driven flows in finite shallow cavities have been investigated for low-Prandtl-number fluids by BenHadid \& Roux (1992). This study has shown the existence of complex flow structures in the form of multicellular steady states and the transition to oscillatory convection has been observed.

Magnetic fields can be used to control these flows of conducting materials. The pioneering experimental work on the action of a constant magnetic field on the flow dynamics for liquid gallium enclosed in a rectangular enclosure was carried out by Hurle et al. (1974). He determined the effect of a constant and uniform magnetic field (horizontal field transverse to the main flow plane) on the threshold conditions and showed that the critical Rayleigh number increases linearly with the square of the Hartmann number. More recent experimental investigations in similar configurations have characterized the braking of the flow by the applied magnetic field (Juel et al. 1999) and the stabilization of the oscillatory instabilities (Hof 2001). Other recent experimental investigations on the action of a constant magnetic field concern convective flows in mercury in a cylindrical cell (Davoust et al. 1999) and the enhancement of heat transfer for natural convection in liquid gallium in a cubical enclosure (Tagawa \& Ozoe 1998a). Theoretical and numerical studies of thermal convection under the action of a constant magnetic field were first conducted for two-dimensional geometries (Oreper \& Szekely 1983, 1984; Kim, Adornato \& Brown 1988; Alboussière, Garandet \& Moreau 1993; BenHadid, Henry \& Kaddeche 1997). For three-dimensional steady flows, results on the effects of a constant magnetic field are given for circular cylinders in BenHadid \& Henry (1996) and for rectangular enclosures in Ozoe \& Okada (1989), and more recently in BenHadid \& Henry (1997), Tagawa \& Ozoe (1998b), Bessaih, Kadja \& Marty (1999), Aleksandrova \& Molokov (2000), and Di Piazza \& Ciofalo (2002). Concerning the action of the magnetic field on time-dependent flows and triggering of instabilities, theoretical and numerical works are very few. By means of linear theory Bojarevics (1995) studied a particular feature of the transition to time-dependent dynamics in a constant magnetic field. His investigation concentrated on the effect of the transverse dimension for a rectangular cavity with infinite longitudinal extent. A recent numerical study (Gelfgat \& Bar-Yoseph 2001) is also devoted to the effect of a magnetic field on the onset of oscillatory instability, but concerns the convective flows in a two-dimensional rectangular cavity.

The aim of this paper is to investigate the effect of a uniform constant magnetic field (either vertical, or horizontal with a direction transverse or longitudinal to the flow) on the stability of buoyant flows of electrically conducting fluid. We focus on the parallel flows induced between infinite horizontal walls by a horizontal temperature gradient. The study is conducted through a numerical linear stability analysis that gives the variation of the critical characteristics of the different types of instabilities when the intensity of the magnetic field is increased. A better understanding of the stabilizing effect due to the applied magnetic fields is then obtained through energy considerations. These results are of fundamental interest in that they show how the magnetic field stabilizes different types of instabilities, but they can also provide information for more realistic finite-length situations.

The plan of the paper is as follows. Next section presents the governing equations of the problem and the basic parallel flows, the stability of which will be studied. The 


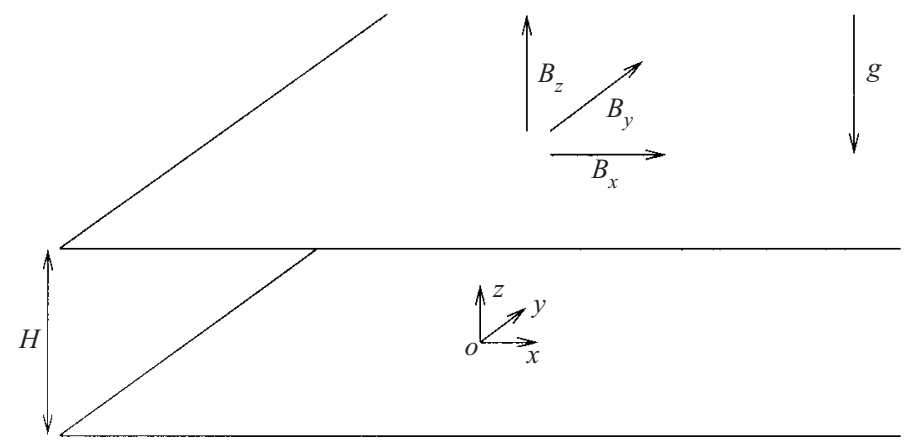

Figure 1. Configuration studied.

numerical approach for the linear stability analysis is then introduced, before the linear stability results for the different orientations of the magnetic field are presented. The information obtained by energy analyses is then given: both as energy balances, but also as energetic contributions to the critical Grashof number. Finally, the conclusion gives a summary of what has been learned in this study and a comparison with results obtained in finite-length cavities.

\section{Governing equations and basic flow}

We consider a viscous electrically conducting fluid with a constant electric conductivity $\sigma_{e}$ contained in a shallow cavity (figure 1) subject to a horizontal temperature gradient $\nabla \tilde{T}$ along $x$ and an external constant magnetic field $\boldsymbol{B}_{0}$. The fluid is assumed to be Newtonian with constant kinematic viscosity $v$ and thermal diffusivity $\kappa$. According to the Boussinesq approximation, the fluid density is considered constant except in the buoyancy term where it is taken as temperature dependent according to the law $\rho=\rho_{0}\left(1-\beta\left(\tilde{T}-\tilde{T}_{0}\right)\right)$ where $\beta$ is the thermal expansion coefficient and $\widetilde{T}_{0}$ a reference temperature. The fluid layer of height $H$ is bounded at the bottom and at the top by rigid surfaces.

In what follows, $G r$ is the Grashof number $\left(G r=g \beta \nabla \tilde{T} H^{4} / \nu^{2}\right)$, Pr the Prandtl number $(P r=v / \kappa)$ and $H a$ the Hartmann number $\left(H a=\left|\boldsymbol{B}_{0}\right| H \sqrt{\sigma_{e} / \rho_{0} v}\right)$.

The magnetic field $\boldsymbol{B}$ is the sum of the applied magnetic field $\boldsymbol{B}_{0}$ and the induced field $\boldsymbol{b}$ such that $\boldsymbol{B}=\boldsymbol{B}_{0}+\boldsymbol{b}$. Since in most laboratory experiments the magnetic Reynolds number $\left(R e_{m}=\mu \sigma_{e} \tilde{V}_{0} H\right.$ where $\mu$ is the magnetic permeability and $\tilde{V}_{0}$ a characteristic velocity) is very small, the magnetic field remains almost unperturbed and the induced magnetic field $\boldsymbol{b}$ is then negligible. To give an indication of how small the magnetic Reynolds number is in experiments, consider a cavity with $H=10^{-2} \mathrm{~m}$ filled with gallium. The electric conductivity is approximately $\sigma_{e}=3.87 \times 10^{6}(\Omega \mathrm{m})^{-1}$. The maximum intensity of the moderate magnetic fields that can be considered is $\left|\boldsymbol{B}_{0}\right|=2 \times 10^{-1} \mathrm{~T}$, giving $H a=88$. For $G r=10^{4}$, the maximum base flow velocity is about $U=0.3 \times 10^{-2} \mathrm{~m} \mathrm{~s}^{-1}$. This leads to a magnetic Reynolds number of $R e_{m} \simeq$ $1.5 \times 10^{-4}$ and an induced magnetic field of approximately $|\boldsymbol{b}| \simeq R e_{m}\left|\boldsymbol{B}_{0}\right| \sim 3 \times 10^{-5} \mathrm{~T}$, which is negligible compared to the value of the applied field $\boldsymbol{B}_{0}$.

We consider $H, H^{2} / v, v / H, \rho_{0} v^{2} / H^{2}, \nabla \tilde{T} H, v\left|\boldsymbol{B}_{0}\right|$ and $\sigma_{e} v\left|\boldsymbol{B}_{0}\right| / H$, as scales for length, time, velocity, pressure, temperature, induced electric potential and induced current, respectively. The dimensionless equations are then

$$
\nabla \cdot \boldsymbol{V}=0
$$




$$
\begin{gathered}
\frac{\partial \boldsymbol{V}}{\partial t}+(\boldsymbol{V} \cdot \nabla) \boldsymbol{V}=-\nabla P+\nabla^{2} \boldsymbol{V}+G r T \boldsymbol{e}_{z}+H a^{2} \boldsymbol{J} \times \boldsymbol{e}_{\boldsymbol{B} 0}, \\
\frac{\partial T}{\partial t}+(\boldsymbol{V} \cdot \nabla T)=\frac{1}{P r} \nabla^{2} T,
\end{gathered}
$$

where the dimensionless variables are the velocity vector $(\boldsymbol{V}=(U, V, W))$, the pressure $P$ and the temperature $T$, and $\boldsymbol{e}_{\boldsymbol{B} 0}$ is the unit vector in the direction of $\boldsymbol{B}_{0}$. In the equation of motion (2.2), the body force $\mathrm{Ha}^{2} \boldsymbol{J} \times \boldsymbol{e}_{\boldsymbol{B} 0}$ is the Lorentz force due to the interaction between the induced electric current density $\boldsymbol{J}$ and the applied magnetic field $\boldsymbol{B}_{0}$. The dimensionless electric current density $\boldsymbol{J}$ is given by Ohm's law for a moving fluid:

$$
\boldsymbol{J}=\boldsymbol{E}+\boldsymbol{V} \times \boldsymbol{e}_{\mathrm{B} 0},
$$

where $\boldsymbol{E}=-\nabla \Phi$ is the dimensionless electric field and $\Phi$ the dimensionless electric potential. Combining the continuity equation for electric current density, $\boldsymbol{\nabla} \cdot \boldsymbol{J}=0$, and Ohm's law (2.4) leads to the equation governing the electric potential, which can be written in dimensionless form as

$$
\nabla^{2} \Phi=\nabla \cdot\left(\boldsymbol{V} \times \boldsymbol{e}_{\boldsymbol{B} 0}\right) .
$$

The mechanical boundary conditions are no-slip conditions at the rigid horizontal boundaries (located at $z=-1 / 2$ and $z=1 / 2$ ). For both the thermal and the electric boundary conditions, limit cases will be considered, either perfectly conducting or perfectly insulating.

Generally, in a real situation, two opposite vertical boundaries (denoted end boundaries) are maintained at different temperatures, $T_{1}$ and $T_{2} \geqslant T_{1}$, creating the horizontal temperature gradient along $x$. This gradient drives the fluid upward near the hot wall and downward near the cold wall, generating a global circulation inside the cavity. In our study dealing with an infinite layer, the cavity is considered to be infinitely long in both horizontal $x$-and $y$-directions. With this assumption, as discussed in previous works by Hart (1983) and Cormack et al. (1974), a stationary parallel flow solution can be found which may approximate the hydrodynamics in a shallow cavity that is not too transversally confined.

In the presence of a constant magnetic field, (2.1)-(2.5) solved for an infinite layer also admit a parallel flow solution. For a vertical magnetic field, this solution is governed by the following equations:

$$
\begin{gathered}
\frac{\partial^{3} U_{0}}{\partial z^{3}}-H a^{2} \frac{\partial U_{0}}{\partial z}-G r=0, \\
\frac{\partial^{2} T_{0}}{\partial z^{2}}=\operatorname{Pr} U_{0} .
\end{gathered}
$$

The solution does not depend on the electric field, but it is dependent on the mechanical and thermal boundary conditions. For a layer between rigid horizontal walls, the analytical expressions for the basic state (similar to those found by Gershuni \& Zhukhovitsky 1976 for a vertical cavity in a horizontal field) are

$$
\begin{gathered}
U_{0}(z)=\frac{G r}{H a^{2}}\left(\frac{\sinh (H a z)}{2 \sinh (H a / 2)}-z\right), \\
T_{0}(x, z)=x+\frac{G r P r}{H a^{2}}\left[\frac{1}{2 H a^{2}} \frac{\sinh (H a z)}{\sinh (H a / 2)}-\frac{z^{3}}{6}+K z\right],
\end{gathered}
$$




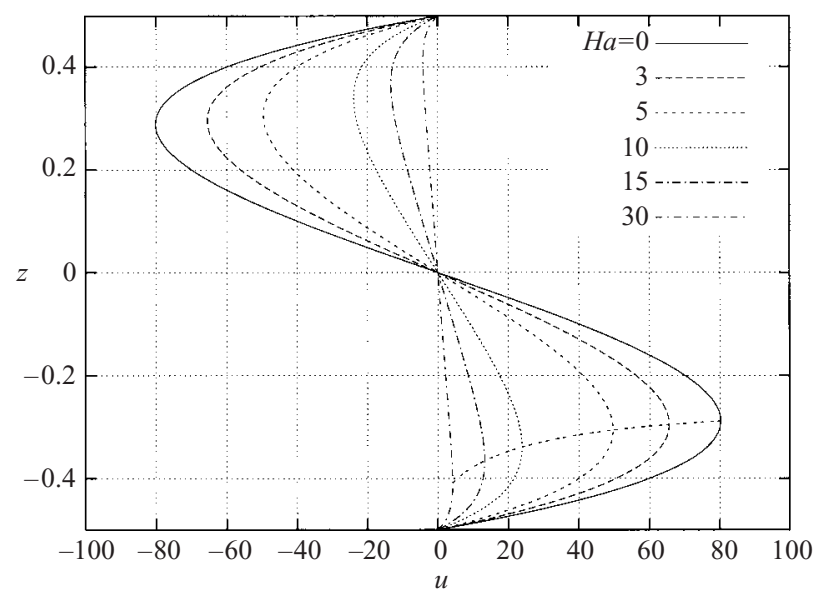

FIGURE 2. Basic velocity profiles for different values of $\mathrm{Ha}\left(\mathrm{Gr}=10^{4}\right)$. A curve is added which follows the maxima of these profiles.

with

$$
K=\left(\frac{1}{24}-\frac{1}{H a^{2}}\right), \quad \text { conducting case }
$$

or

$$
K=\left(\frac{1}{8}-\frac{\cosh (H a / 2)}{2 H a \sinh (H a / 2)}\right), \quad \text { insulating case. }
$$

The corresponding velocity profiles are given in figure 2 for $G r=10^{4}$ and for different values of $\mathrm{Ha}$ up to $\mathrm{Ha}=30$. This solution is valid in the core of a finite-length cavity, and also representative of the flow in the cavity for not too high a vertical magnetic field. On the other hand, for a sufficiently high field, jets will develop in the parallel layers along the lateral boundaries and they generally will be more unstable than the core flow.

In the case of a horizontal longitudinal magnetic field (the magnetic field is applied along the $x$-direction), there is no direct effect of the field on the parallel flow in the layer as the velocity $\left(U_{0} \boldsymbol{e}_{x}\right)$ is parallel to the field direction $\left(U_{0} \boldsymbol{e}_{x} \times \boldsymbol{e}_{\boldsymbol{B} 0}=0\right)$. The basic flow is then the flow without magnetic field (the limit of equations (2.8)-(2.11) for $H a \rightarrow 0$ ), i.e.

$$
\begin{gathered}
U_{0}(z)=\frac{G r}{24}\left(4 z^{3}-z\right), \\
T_{0}(x, z)=x+\frac{G r P r}{5760}\left(48 z^{5}-40 z^{3}+7 z\right), \quad \text { conducting case, } \\
T_{0}(x, z)=x+\frac{G r P r}{5760}\left(48 z^{5}-40 z^{3}+15 z\right), \quad \text { insulating case. }
\end{gathered}
$$

This parallel flow without the influence of the magnetic field can be obtained in a finite-length cavity beyond a distance $x \sim H a$ from the end boundaries (Alboussière et al. 1997). This distance is the length necessary for the parallel layers developing along the horizontal walls to merge $\left(\delta_{\|} \sim \mathrm{Ha}^{-1 / 2} x^{1 / 2}\right.$, where $\delta_{\|}$is the dimensionless parallel layer thickness). Therefore, solution (2.12)-(2.14) will be valid in the core of a finite-length cavity only if the cavity dimension along $x$ is $\gg H a$. To observe this 
parallel flow solution the cavity must then be quite long in the $x$-direction and $\mathrm{Ha}$ not too large.

In the case of a horizontal transverse magnetic field (the magnetic field is applied along the $y$-direction), the basic parallel flow in the layer is still the flow without magnetic field given by equations (2.12)-(2.14). Indeed, a transverse magnetic field has a direct action on the fluid flow along both the $x$ - and $z$-directions, which are the directions of main flow in the cavity. But in two-dimensional situations, it is well known that, due to the electric potential effect, the electric current and consequently the Lorentz force vanish in the whole cavity, finally giving no effect of the field on the flow. In fact, in finite-length cavities, the basic flow is generally different for a transverse field (BenHadid \& Henry 1994; Aleksandrova \& Molokov 2000). The reason for this difference is the three-dimensional character of the real flow without magnetic field due to the no-slip condition at the lateral walls. Under a magnetic field, the flow is in fact braked, becomes more two-dimensional and active Hartmann layers develop at the lateral walls.

\section{Numerical approach}

The stability of the basic flow solution ((2.8)-(2.11) or (2.12)-(2.14)) is investigated here in a general way by a linear analysis. The solution of the three-dimensional problem is written as

$$
(\boldsymbol{V}, P, T, \Phi)=\left(\boldsymbol{V}_{0}, P_{0}, T_{0}, \Phi_{0}\right)+(\boldsymbol{v}, p, \theta, \phi)
$$

i.e. the sum of the basic flow quantities and perturbations. Substitution into equations (2.1)-(2.5) and linearization with respect to the perturbations yields

$$
\begin{gathered}
\nabla \cdot \boldsymbol{v}=0, \\
\frac{\partial \boldsymbol{v}}{\partial t}+\left(\boldsymbol{V}_{0} \cdot \nabla\right) \boldsymbol{v}+(\boldsymbol{v} \cdot \nabla) \boldsymbol{V}_{0}=-\nabla p+\nabla^{2} \boldsymbol{v}+G r \theta \boldsymbol{e}_{z}+H a^{2}\left(-\nabla \phi+\boldsymbol{v} \times \boldsymbol{e}_{\boldsymbol{B} 0}\right) \times \boldsymbol{e}_{\boldsymbol{B} 0}, \\
\frac{\partial \theta}{\partial t}+\boldsymbol{V}_{0} \cdot \nabla \theta+\boldsymbol{v} \cdot \nabla T_{0}=\frac{1}{P r} \nabla^{2} \theta, \\
\nabla^{2} \phi=\boldsymbol{e}_{\boldsymbol{B} 0} \cdot(\nabla \times \boldsymbol{v}),
\end{gathered}
$$

where $\boldsymbol{V}_{0}=\left(U_{0}, 0,0\right)$ and $\boldsymbol{e}_{\boldsymbol{B} 0}$ is the direction of the magnetic field (i.e. $\boldsymbol{e}_{z}$ when the field is vertical, $\boldsymbol{e}_{y}$ when it is transverse, and $\boldsymbol{e}_{x}$ when it is longitudinal).

Only boundary conditions in the $z$-direction (for $z=-1 / 2$ and $z=1 / 2$ ) are needed because we will use periodic disturbances in the horizontal $x$ - and $y$-directions. These conditions are:

no-slip boundary conditions: $\boldsymbol{v}=0$;

conducting thermal boundary conditions: $\theta=0$, or adiabatic thermal boundary conditions: $\partial \theta / \partial z=0$;

conducting electric boundary conditions: $\phi=0$, or insulating electric boundary conditions: $\boldsymbol{j} \cdot \boldsymbol{e}_{z}=0$.

The linear stability study consists, for fixed values of the Hartmann number $\mathrm{Ha}$ and Prandtl number $P r$, of the determination of $G r_{c}$, the critical value of $G r$ beyond which the basic flow loses its stability. For the normal modes analysis, the set of equations (3.2)-(3.5) is transformed using the following disturbances:

$$
(\boldsymbol{v}, p, \theta, \phi)=(\boldsymbol{v}, p, \theta, \phi)(z) \mathrm{e}^{\mathrm{i}(h x+k y)+\omega t},
$$


where $h$ and $k$ are real wavenumbers in the longitudinal, $x$, and transverse, $y$, directions, respectively, and $\omega=\omega_{r}+\mathrm{i} \omega_{i}$ is a complex eigenvalue. The real part of $\omega$ represents an amplification rate and its imaginary part an oscillation frequency. An eigenvalue problem is obtained: $L X=\omega M X$, where $X=(v(z), p(z), \theta(z), \phi(z))$, $L$ is a linear operator depending on $h, k, P r, G r$ and $H a$, and $M$ is a constant linear operator. This generalized eigenvalue problem is solved with the spectral Tau Chebyshev method by means of a numerical procedure using the QZ eigenvalue solver of the NAG library. From the thresholds $\mathrm{Gr}_{0}(\mathrm{Pr}, \mathrm{Ha}, h, k)$ (values of $\mathrm{Gr}$ for which an eigenvalue has a real part equal to zero whereas all the other eigenvalues have negative real parts), the critical Grashof number $G r_{c}$ can be obtained after minimization along $h$ and $k\left(G r_{c}=\mathrm{in} f_{(h, k) \in \mathscr{R ^ { 2 }}} G r_{0}(\mathrm{Pr}, \mathrm{Ha}, h, k)\right)$.

As already mentioned in the introduction, previous works have shown that for rigid boundaries and small $P r$ without a magnetic field the two instabilities which can affect the flow in the layer are either longitudinal or transverse instabilities, corresponding respectively to $h=0$ and $k=0$. Laure \& Roux (1987) mentioned that due to the symmetry properties existing for rigid boundaries, $G r_{0}$ is an even function of $h$ and $k$. This implies that a minimum can be found for $(h, 0)$ or $(0, k)$. They have verified that two local minima of $G r_{0}$ are of this type and that they are the only minima of $G r_{0}$ for small $\mathrm{Pr}$.

Our objective in this work was to examine the action of the magnetic field on these two instabilities, which are the most important for small-Pr fluids in a layer between rigid boundaries. $G r_{c}$ was then obtained for perturbations where either $h$ or $k$ is equal to zero. We have verified that with magnetic field also, for low $\mathrm{Pr}$ the same types of instabilities with $h$ or $k$ equal to zero were found and that there was no other minimum for modes with both $h \neq 0$ and $k \neq 0$.

The critical Grashof numbers were determined by expanding the variables in the $z$-direction in a Chebyshev series with 20 to 30 collocation points. This was sufficient for an accurate determination of the linear stability characteristics. Except where otherwise stated, the results have been obtained for insulating electric boundary conditions.

\section{Linear stability results}

\subsection{Vertical magnetic field}

The action of the vertical magnetic field on the two-dimensional and three-dimensional instabilities is shown in figure $3(a, b)$ through neutral stability curves. These curves give the evolution of the critical Grashof number $\mathrm{Gr}_{c}$ as a function of $\mathrm{Pr}$ for different Hartmann numbers with thermal boundary conditions either conducting (figure $3 a$ ) or insulating (figure $3 b$ ). Both figures give qualitatively similar results. A first observation is that an increase of $\mathrm{Ha}$ shifts the onset of the instabilities to higher Grashof numbers (stabilizing effect of the magnetic field), this effect appearing more efficient for the two-dimensional instabilities. Concerning the dependence on the Prandtl number, we find the usual behaviour. For the two-dimensional stationary modes, on increasing $\mathrm{Pr}$, the critical Grashof number first remains almost constant (at least up to $\operatorname{Pr} \sim 0.01$ for $H a \leqslant 5)$ and then starts to increase, whereas for the three-dimensional oscillatory modes, $G r_{c}$ undergoes a rapid decrease as $\mathrm{Pr}$ is increased $\left(\mathrm{Pr}^{-1}\right.$ dependence), then reaches a minimum before beginning to increase. The main difference that appears when $\mathrm{Ha}$ is increased is that for both modes the observed increase occurs for smaller values of $\mathrm{Pr}$. We recall that the difference in behaviours obtained in the small-Pr limit (constant value or strong increase as $P r \rightarrow 0$ ) indicates that the two-dimensional 

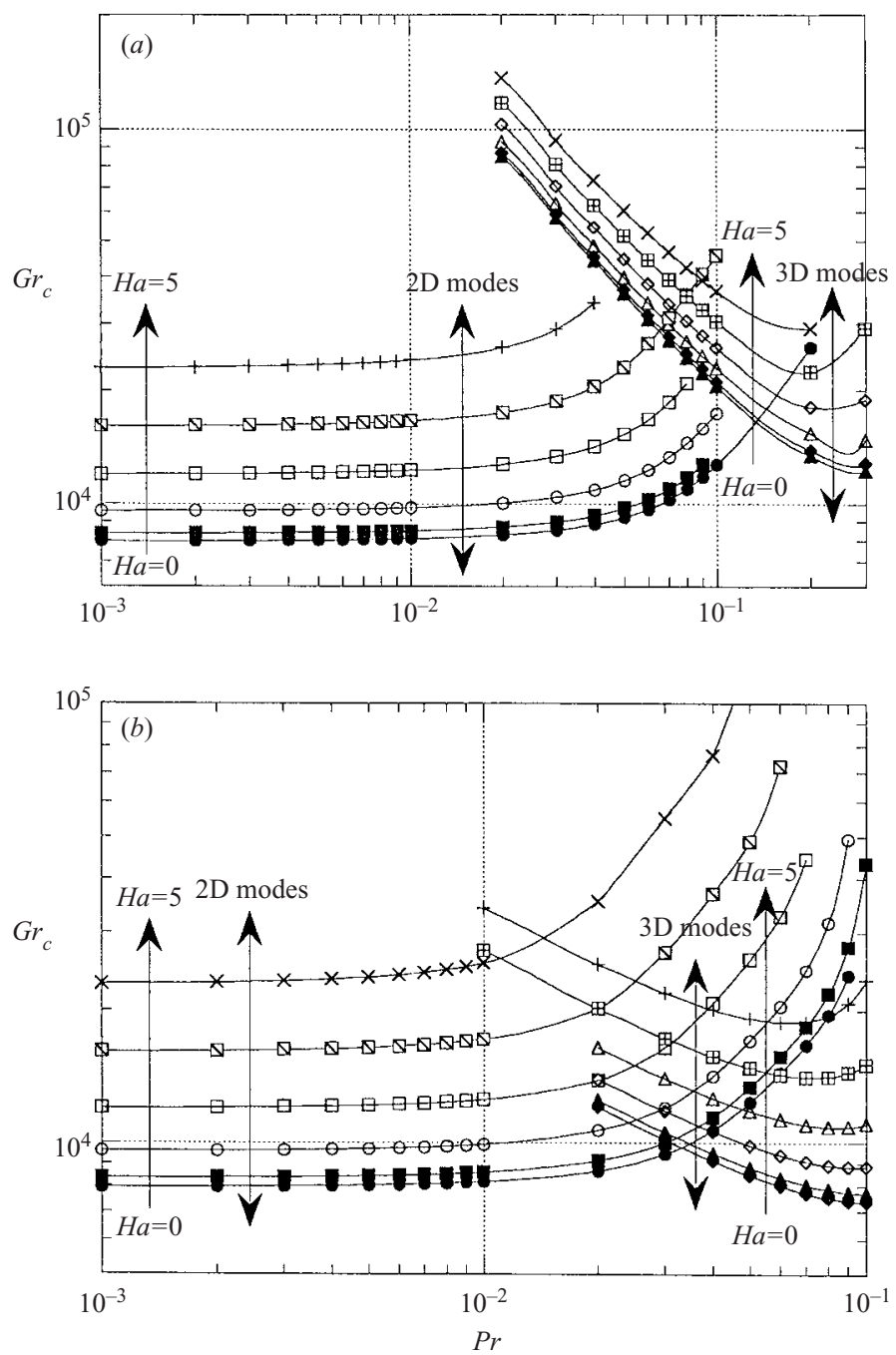

FiguRE 3. Variation of the thresholds $G r_{c}$ as a function of $\mathrm{Pr}$ for two-dimensional and three-dimensional instabilities and different values of $H a$ (vertical magnetic field). (a) Thermally conducting boundaries; $(b)$ thermally insulating boundaries.

instability is of dynamical origin whereas the three-dimensional instability is of thermal origin.

From figure $3(a, b)$, it can also be seen that for any $H a$, the curves for the two-dimensional and three-dimensional instabilities intersect at a certain value of the Prandtl number, denoted $\operatorname{Pr}_{T}$. As shown in figure 4, $\operatorname{Pr}_{T}$ is found to decrease quickly with $H a$, in connection with the fact that the two-dimensional modes are better stabilized than the three-dimensional modes. This implies that the domain where two-dimensional modes are critical shrinks when the magnetic field is applied, corresponding for example for the insulated case to $\mathrm{Pr} \leqslant 0.001$ at $\mathrm{Ha}=10$ to be compared to $\mathrm{Pr} \leqslant 0.034$ at $\mathrm{Ha}=0$. As a consequence, for gallium experiments $(\operatorname{Pr}=0.026)$ as in Hurle et al. (1974), longitudinal rolls would be the expected outcome once $\mathrm{Ha} \geqslant 3$. 


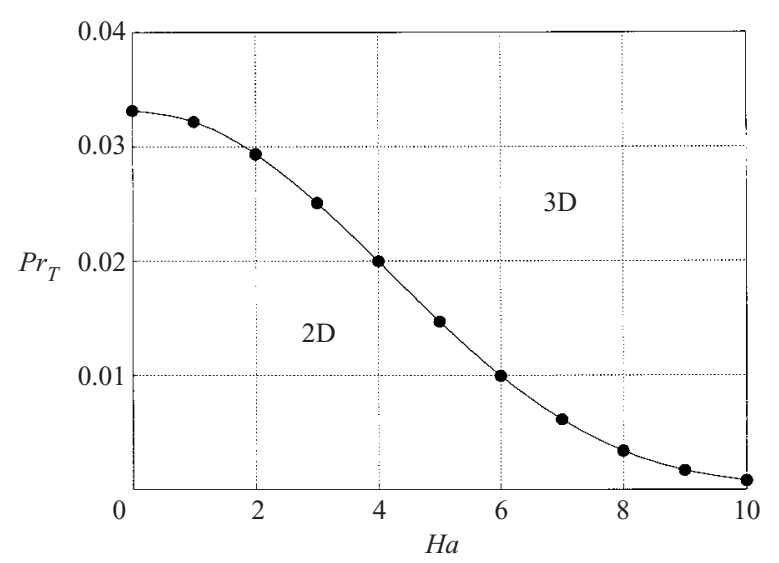

FIGURE 4. Variation of $\operatorname{Pr}_{T}$ (transition Prandtl number between the critical domains for two-dimensional and three-dimensional instabilities) as a function of $\mathrm{Ha}$ (vertical magnetic field, case with thermally insulating boundaries).

The wavenumbers of the instabilities are given in figure 5 as a function of the Prandtl number and for different Hartmann numbers. In the case of the two-dimensional instability (figure $5 a$ ), it is found that the magnetic field induces a decrease of the wavenumber $h_{c}$ (increase of the size of the marginal cells), also causing the decrease of the wavenumber with $\mathrm{Pr}$ to occur at smaller $\mathrm{Pr}$ values. In the case of the threedimensional instability, the result is opposite, with an increase of the wavenumber $k_{c}$ when $\mathrm{Ha}$ is increased. This last result is in agreement with what is obtained in the Rayleigh-Bénard situation (decrease of the size of the marginal cells, see Chandrasekhar 1961) where the instabilities are also of thermal origin. The frequency $f_{c}$ of the oscillatory three-dimensional instabilities is shown in figure 6 to increase with $\mathrm{Ha}$.

The stabilizing effect of the vertical magnetic field on the different instabilities is more precisely characterized for the thermally conducting case in figure 7 where the instability thresholds are given as a function of $\mathrm{Ha}$ for different $\mathrm{Pr}$, each instability being shown up to the limit $\mathrm{Ha}$ value where it can be found. From figure 7, the critical Grashof number $G r_{c}$ is found to vary exponentially with $H a^{2}$ for the two-dimensional instabilities (for example $G r_{c}=G r_{c_{0}} \exp \left(0.04 H a^{2}\right)$ for $\operatorname{Pr}=0.001$ and $G r_{c}=G r_{c_{0}} \exp \left(0.08 \mathrm{Ha}^{2}\right)$ for $\left.\mathrm{Pr}=0.1\right)$ and as $\mathrm{Ha}^{2}$ for the threedimensional instabilities (for example $G r_{c}-G r_{c_{0}}=G r_{c_{0}} 0.0252 \mathrm{Ha}^{2}$ for $\mathrm{Pr}=0.02$ and $G r_{c}-G r_{c_{0}}=G r_{c_{0}} 0.0306 \mathrm{Ha}^{2}$ for $\mathrm{Pr}=0.1$, with $G r_{c_{0}}$ being the corresponding value of $\mathrm{Gr}_{c}$ at $\mathrm{Ha}=0$ ). These correlations are quite good for low Hartmann numbers (roughly up to $H a=10$ ) but differences appear for larger values of $H a$ (for example a systematic value above the correlations is found for the three-dimensional thresholds). In any case, they clearly confirm that the vertical magnetic field has a more significant stabilizing effect on the two-dimensional instabilities than on the three-dimensional instabilities. Note that for the two-dimensional instabilities the electric potential $\phi$ vanishes in the whole cavity as the conservation of current is automatically implied by the conservation of mass.

Figure 7 also indicates that there are limit $H a$ values (denoted $H a_{l}$ and depending on $P r$ ) for the existence of both instabilities. For the two-dimensional instabilities, the value of $H a_{l}$ has been estimated for different $\operatorname{Pr}$ (integer value of $\mathrm{Ha}$ just before the disappearance of the instability): $H a_{l}=6$ for $\operatorname{Pr}=0.1, H a_{l}=11$ for $\operatorname{Pr}=0.02$, 

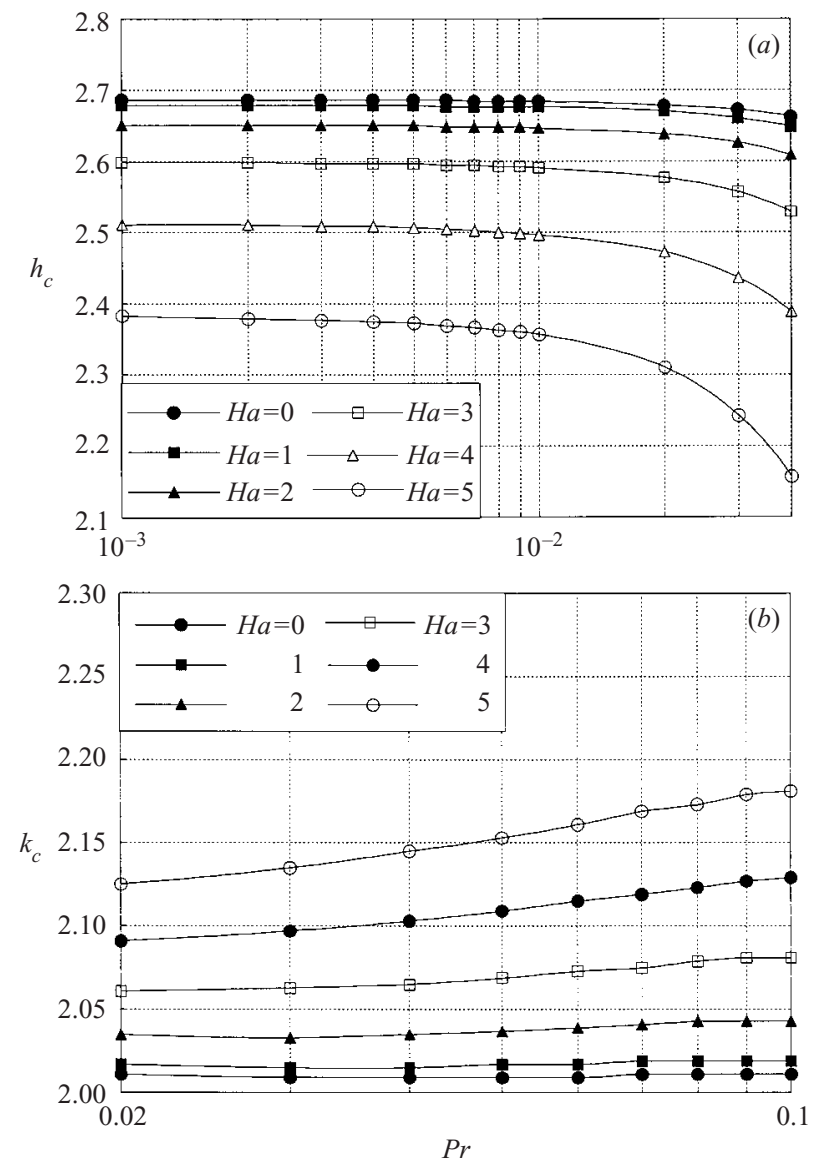

FIGURE 5. Variation of the critical wavenumbers of the instabilities as a function of $\operatorname{Pr}$ for different values of $\mathrm{Ha}$ (vertical magnetic field, case with thermally conducting boundaries). (a) Wavenumber $h_{c}$ for the two-dimensional instabilities; $(b)$ wavenumber $k_{c}$ for the three-dimensional instabilities.

and $H a_{l}=14$ for $\mathrm{Pr}=0.001$. An illustration of how the two-dimensional instability disappears is given in figure 8 for $\mathrm{Pr}=0.001$ in a graph showing the thresholds $G r_{0}$ as a function of the wavenumber $h$. The clear minimum of the curves (corresponding to $\mathrm{Gr}_{c}$ ) obtained for small $\mathrm{Ha}$ becomes less pronounced and evolves towards smaller values of $h$ when $H a$ is increased, before disappearing between $H a=14$ and $H a=15$. These results can also be interpreted in terms of limit $\operatorname{Pr}$ values (denoted $P r_{l}$ ) for the two-dimensional instability as is suggested in figure 3 by the strong increase of the thresholds as $\mathrm{Pr}$ increases: $\mathrm{Pr}_{l}$ will evolve from values around 0.25 for $\mathrm{Ha}=0$ to values around 0.001 for $H a=14$, showing a strong shrinkage of the existence domain of the two-dimensional instabilities as $\mathrm{Ha}$ is increased. For the three-dimensional instabilities, two values of $H a_{l}$ have been estimated: $H a_{l}=12$ for $\operatorname{Pr}=0.1$ and $H a_{l}=32$ for $\mathrm{Pr}=0.02$. In terms of the existence domain of the three-dimensional instabilities, the shrinkage with $\mathrm{Ha}$ is less effective than for the two-dimensional instabilities, $P r_{l}$ evolving from values around 0.4 for $H a=0$ to values around 0.02 for $H a=32$. The scenario of the disappearance of the three-dimensional instabilities is completely different; it is illustrated in figure $9(a, b)$ for $\operatorname{Pr}=0.1$ through two graphs 


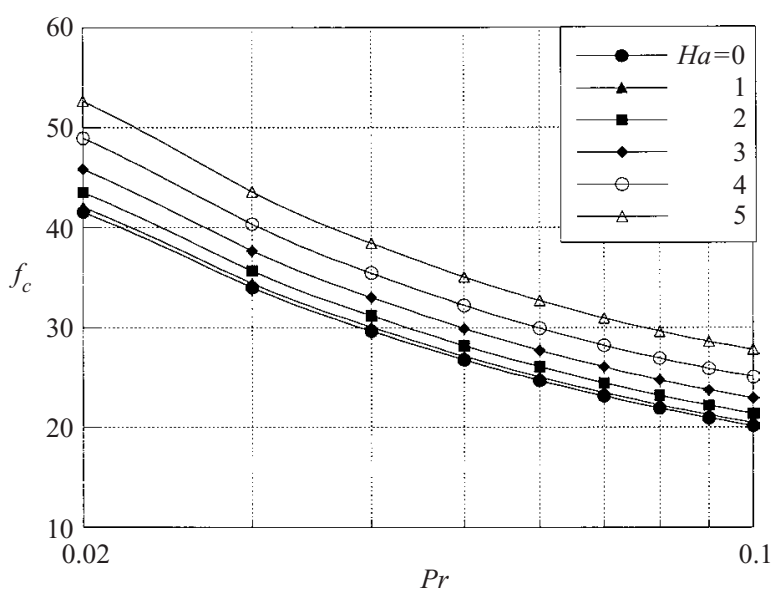

FIGURE 6. Variation of the critical frequency as a function of $\operatorname{Pr}$ for the three-dimensional oscillatory instabilities and different values of $\mathrm{Ha}$ (vertical magnetic field, case with thermally conducting boundaries).

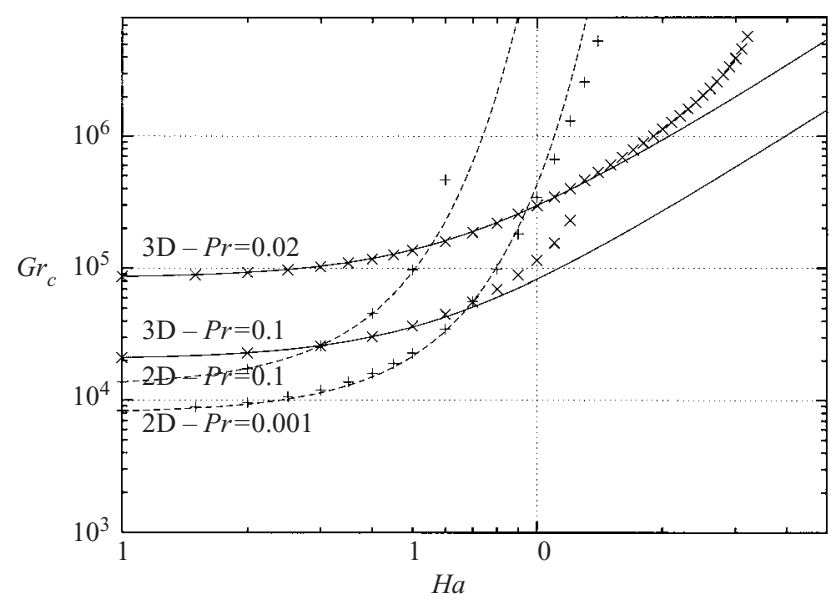

FIGURE 7. Variation of the thresholds $G r_{c}$ as a function of $H a$ for two-dimensional and three-dimensional instabilities and different values of $\operatorname{Pr}$ (vertical magnetic field, case with thermally conducting boundaries). The points correspond to the calculated results and the lines to well-correlated characteristic laws given in the text.

giving the threshold $G r_{0}$ as a function of $k$ for $H a=12$ and the real part of the leading eigenvalue (larger real part) as a function of $G r$ for $k=1.6$ and different $H a$. The eigenvalue corresponding to the three-dimensional instability evolves as a function of $\mathrm{Gr}$ as a bell curve (figure $9 b$ ). The maximum of this curve, which is positive for $H a=0$, decreases as $H a$ is increased and becomes negative beyond a certain value of $\mathrm{Ha}$ leading to the suppression of the critical transition for this three-dimensional mode. Figure $9(a, b)$ shows that for $H a=12$ there is an isolated domain of instability which will finally disappear for a slightly higher $H a$. This type of behaviour which leads to hysteresis phenomena can also be found in Gelfgat \& Bar-Yoseph (2001) for two-dimensional rectangular cavities. The fact that the instability disappears through a maximum of the eigenvalue curve (horizontal tangent in figure $9 b$ ) explains the 


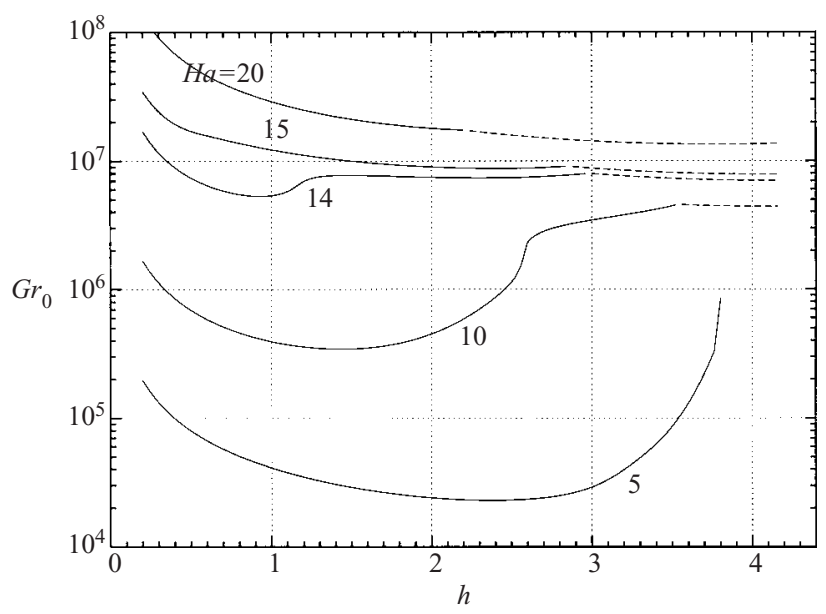

FiguRE 8. Disappearance of the two-dimensional instabilities for a vertical magnetic field. Variation of the threshold $\mathrm{Gr}_{0}$ as a function of the wavenumber $h$ for different values of $\mathrm{Ha}$ (case with thermally conducting boundaries, $P r=0.001$ ).

strong increase in the thresholds found for the three-dimensional instability before its disappearance. All these results on the disappearance of the instabilities when $\mathrm{Ha}$ is increased are summarized in figure 10 by curves in a $(\mathrm{Pr}, \mathrm{Ha})$-plane, giving rough approximations of the limits of existence of the two-dimensional and threedimensional instabilities. It is clear from this figure that the domain of existence of both instabilities diminishes, but that two-dimensional instabilities are much more affected.

To conclude this part, we make some general comments about the boundary conditions. The influence of the thermal boundary conditions may be summarized as follows: $\left(G r_{c}\right)_{\text {conducting }}<\left(G r_{c}\right)_{\text {insulated }}$ for the two-dimensional instabilities and $\left(G r_{c}\right)_{\text {conducting }}>\left(G r_{c}\right)_{\text {insulated }}$ for the three-dimensional instabilities. This can be explained by the fact that for the two-dimensional instabilities of dynamical origin the temperature fluctuations which are favoured by the insulated conditions stabilize the flow. On the contrary, for the three-dimensional instabilities of thermal origin the temperature fluctuations destabilize the flow. A consequence is that the value of $\operatorname{Pr}_{T}$ is smaller in the insulated case than in the conducting case. The electrical boundary conditions have no effect on the two-dimensional instabilities for which the electric potential $\phi$ plays no role $(\phi=0$ everywhere). For the three-dimensional instabilities, it has been found that electrically conducting boundary conditions are more stabilizing, which can be explained by the fact that potential effects which limit the stabilization are less important in this case as loops of current can close in the conducting boundaries. This improved stabilization still corresponds to characteristic laws for the critical thresholds varying as $G r_{c}-G r_{c_{0}} \sim H a^{2}$.

\subsection{Horizontal magnetic field}

\subsubsection{Transverse magnetic field}

The transverse magnetic field only has an effect on the three-dimensional instabilities. The two-dimensional instabilities are not affected by the transverse field which is perpendicular to the plane of these instabilities because the electric current and the Lorentz force vanish in the whole cavity, as it was the case for the basic 

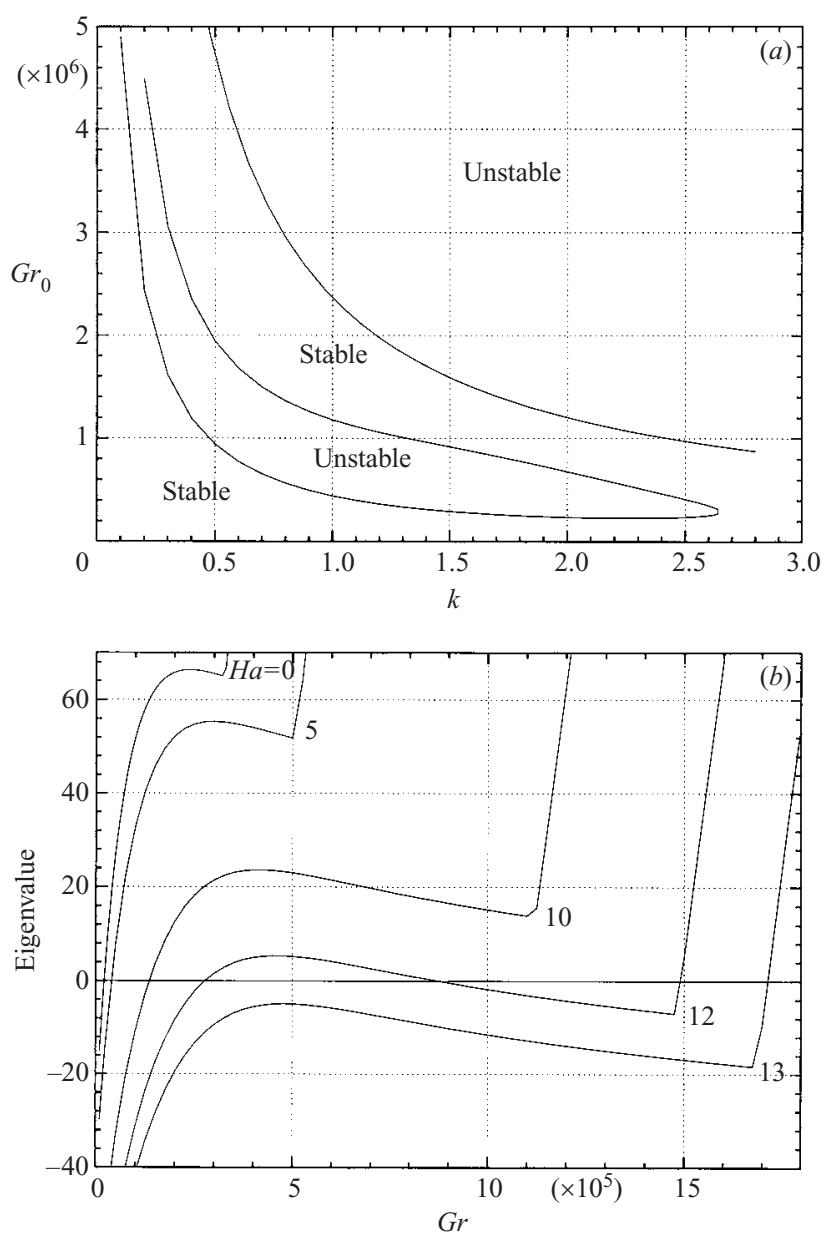

FIGURE 9. Disappearance of the three-dimensional instabilities for a vertical magnetic field (case with thermally conducting boundaries, $P r=0.1$ ). (a) Variation of the threshold $G r_{0}$ as a function of the wavenumber $k$ for $H a=12 ;(b)$ variation of the real part of the leading eigenvalue as a function of $\mathrm{Gr}$ for $k=16$ and different values of $\mathrm{Ha}$.

flow (see $\$ 2$ ). The thresholds for these two-dimensional instabilities thus remain those obtained for $H a=0$ (figure $3 a, b$ ).

The results for the three-dimensional oscillatory instabilities are given in figures 1113. The stability diagram is presented in the $G r_{c}-H a$ parameter space for the range $0.005 \leqslant P r \leqslant 0.1$ (figure 11). It is found that the stabilization by the transverse magnetic field is less efficient than by the vertical field (small variations of $G r_{c}$ in the small-Ha domain), but it occurs up to large $H a$ (no limit $H a$ values) with an asymptotic increase $\mathrm{Gr}_{c} \sim \mathrm{Ha}$ beyond a certain value of $\mathrm{Ha}$, this value increasing roughly from 20 to 60 as $\mathrm{Pr}$ decreases from 0.1 to 0.005 . The dependence on $\mathrm{Pr}$ is globally not much changed when $\mathrm{Ha}$ is increased and is close to that found for $\mathrm{Ha}=0\left(\mathrm{Pr}^{-1}\right.$ dependence for small $\left.\mathrm{Pr}\right)$.

The wavenumber $k_{c}$ (figure 12) also remains almost constant for small $\mathrm{Ha}$ before decreasing asymptotically as $\mathrm{Ha}^{-1}$ for larger values. Note that unlike the case of a vertical magnetic field, it is found here that the size of the marginal cells for these 


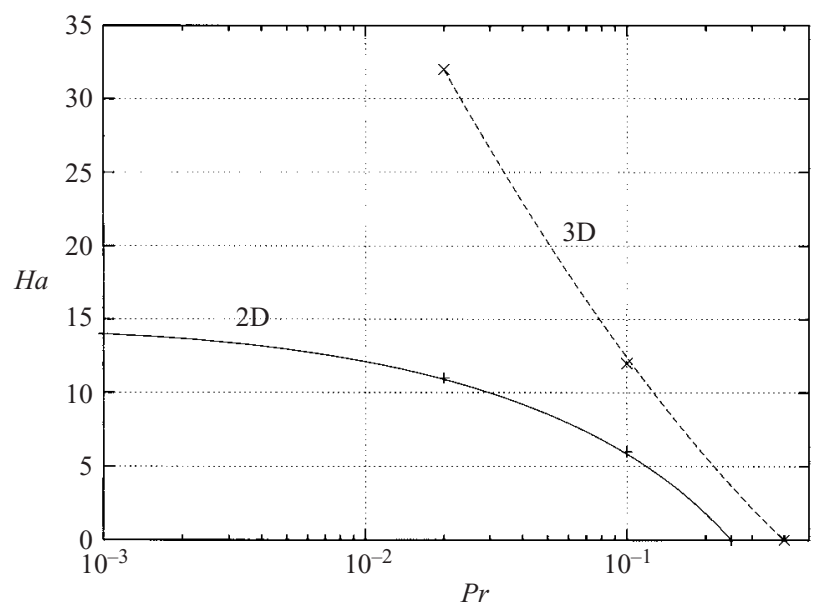

FIGURE 10. Limits of existence of the two-dimensional and three-dimensional instabilities in a $(\mathrm{Pr}, \mathrm{Ha})$-plane (vertical magnetic field, case with thermally conducting boundaries).

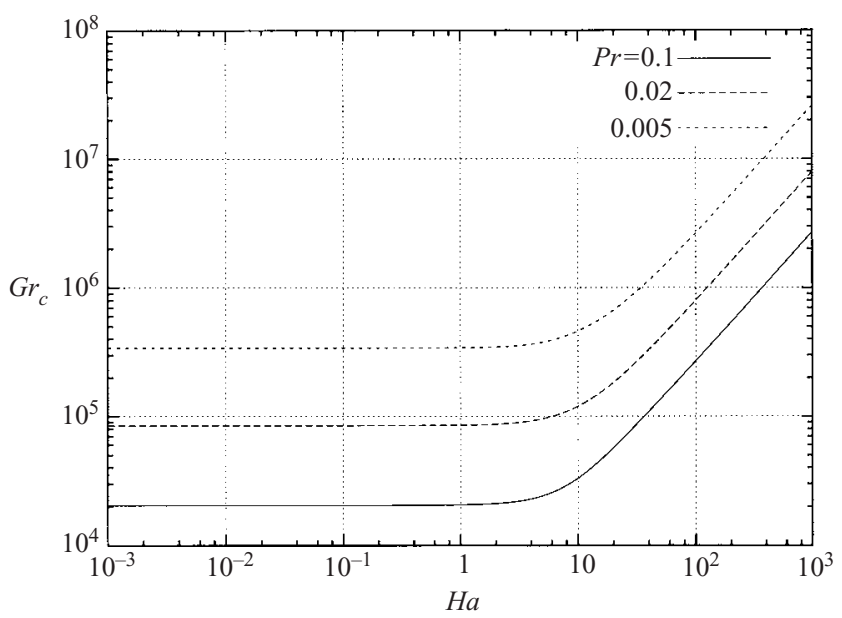

FiguRE 11. Variation of the threshold $G r_{c}$ as a function of $H a$ for the three-dimensional instabilities and different values of $\operatorname{Pr}$ (horizontal transverse magnetic field, case with thermally conducting boundaries).

three-dimensional instabilities increases with $H a$, in agreement with the expected effect of lengthening of the convective cells in the direction of the magnetic field. Finally, the frequency of these three-dimensional instabilities also presents different domains as $\mathrm{Ha}$ is increased (figure 13): it remains almost constant for small $\mathrm{Ha}$, then increases significantly beyond $H a=1$, before reaching a constant asymptotic value for large $H a$. The plateau values and the length of the increase zone depend quite strongly on Pr.

\subsubsection{Longitudinal magnetic field}

The longitudinal magnetic field only has an effect on the two-dimensional instabilities. The three-dimensional instabilities are not affected by the longitudinal field, because the field is perpendicular to the main plane of these instabilities (there is 


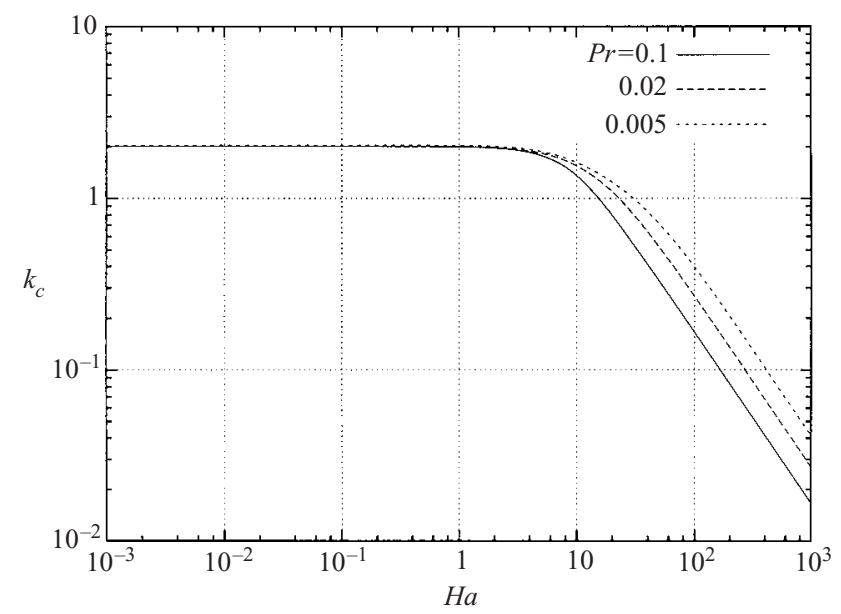

FIGURE 12. Variation of the wavenumber $k_{c}$ as a function of $H a$ for the three-dimensional instabilities and different values of $\operatorname{Pr}$ (horizontal transverse magnetic field, case with thermally conducting boundaries).

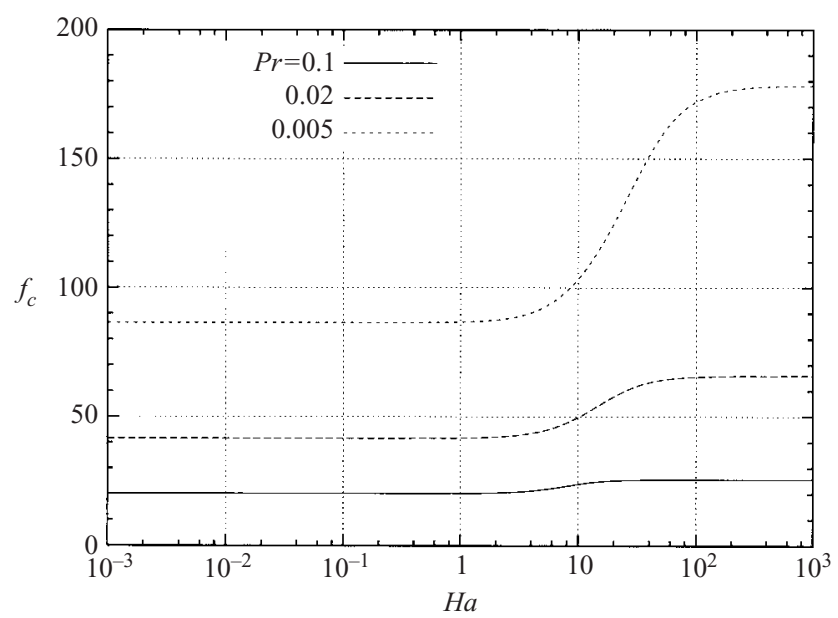

FIGURE 13. Variation of the frequency $f_{c}$ as a function of $H a$ for the three-dimensional instabilities and different values of $\operatorname{Pr}$ (horizontal transverse magnetic field, case with thermally conducting boundaries).

no electric current in this yoz-plane) and is parallel to the other velocity component along $x$ (no current induced by this component). The thresholds for these threedimensional instabilities thus remain those obtained for $H a=0$ (figure $3 a, b$ ).

The results for the two-dimensional stationary instabilities in a longitudinal magnetic field (given in figures 14 and 15) look qualitatively similar to those obtained for the three-dimensional instabilities in a transverse field. As $\mathrm{Ha}$ is increased, $G r_{c}$ and the wavenumber $h_{c}$ remain almost constant for small $H a$ and then show asymptotic behaviours beyond $\mathrm{Ha}=20, \mathrm{Gr}_{c}$ increasing as $\mathrm{Ha}$ and $h_{c}$ decreasing as $\mathrm{Ha}^{-1}$. The variation of $h_{c}$ still corresponds to the lengthening of the convective cell in the direction of the magnetic field. The dependence on $\mathrm{Pr}$ is mainly characterized by constant values for small $\mathrm{Pr}$. For larger $\mathrm{Pr}$, the dependence of $G r_{c}$ seems not much 


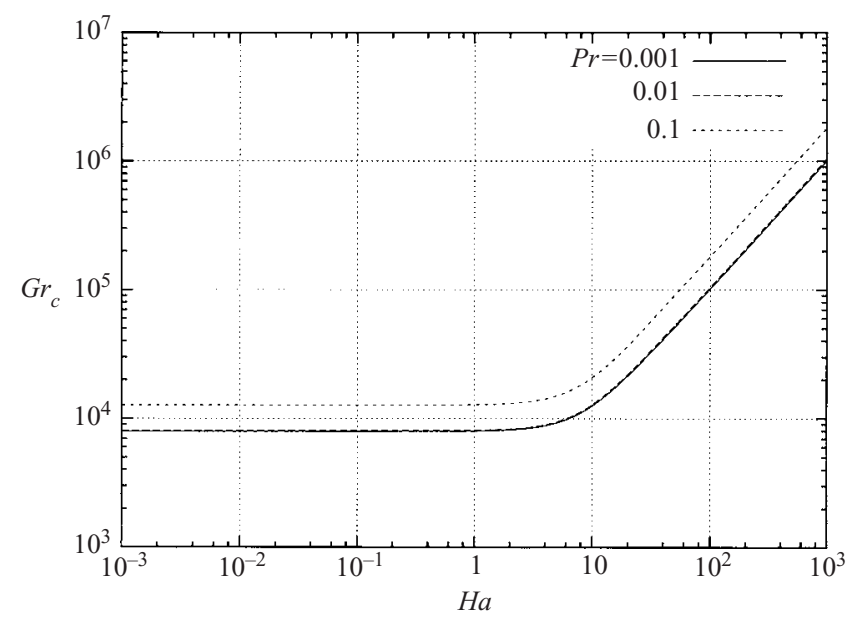

FIGURE 14. Variation of the threshold $G r_{c}$ as a function of $H a$ for the two-dimensional instabilities and different values of $\operatorname{Pr}$ (horizontal longitudinal magnetic field, case with thermally conducting boundaries).

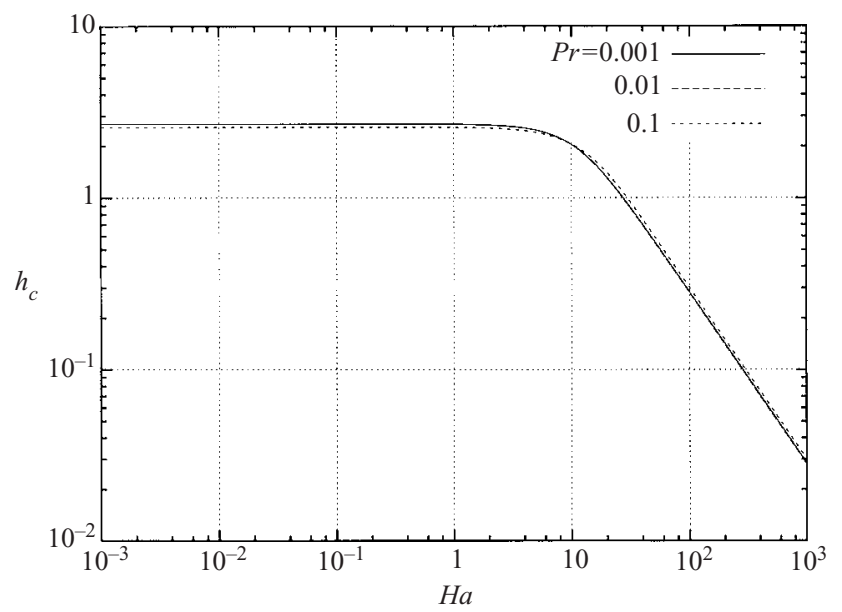

FIGURE 15. Variation of the wavenumber $h_{c}$ as a function of $H a$ for the two-dimensional instabilities and different values of $\operatorname{Pr}$ (horizontal longitudinal magnetic field, case with thermally conducting boundaries).

changed as $H a$ is increased, whereas that of $h_{c}$ (which is small) presents an inversion beyond $H a=10$. Finally, note that the vertical magnetic field was found to be more efficient in stabilizing these two-dimensional instabilities.

\section{Energy analyses}

A deeper physical understanding of the instability mechanisms and of the stabilization effects in these situations with an applied magnetic field can be gained by energy analyses. From the linear stability equations (3.3) and (3.4), energy equations for the fluctuating kinetic energy and thermal energy can be derived: equation (3.3) is multiplied by $\boldsymbol{v}^{*}$, the complex conjugate of $\boldsymbol{v}$, equation (3.4) by $\theta^{*}$, and after 
integration along $z$, the real parts of the resulting equations are taken ( $R e$ is used to denote the real part). Having in mind that $v$ and $\theta$ are perturbations satisfying (3.6) and after some transformations to eliminate zero terms such as the pressure integral (cases with no inflow or outflow), the following relationships for the rate of change of the fluctuating kinetic energy $K$ and the fluctuating thermal energy $\Theta$ are then obtained:

$$
\operatorname{Re}\left(\frac{\partial K}{\partial t}\right)=\omega_{r} \int_{z} v v^{*} \mathrm{~d} z=K_{f}+K_{d}+K_{b}+K_{m},
$$

with

$$
K_{f}=-\operatorname{Re}\left(\int_{z} w \frac{\partial U_{0}}{\partial z} u^{*} \mathrm{~d} z\right)
$$

the production of fluctuating kinetic energy by shear of mean flow,

$$
K_{d}=\operatorname{Re}\left(\int_{z} \nabla^{2} \boldsymbol{v} \boldsymbol{v}^{*} \mathrm{~d} z\right)
$$

the viscous dissipation of fluctuating kinetic energy,

$$
K_{b}=\operatorname{Re}\left(G r \int_{z} \theta w^{*} \mathrm{~d} z\right)
$$

the production of fluctuating kinetic energy by buoyancy forces,

$$
K_{m}=\operatorname{Re}\left(H a^{2} \int_{z}\left[\left(-\nabla \phi+\boldsymbol{v} \times \boldsymbol{e}_{\boldsymbol{B} 0}\right) \times \boldsymbol{e}_{\boldsymbol{B} 0}\right] \boldsymbol{v}^{*} \mathrm{~d} z\right)
$$

the dissipation of fluctuating kinetic energy by magnetic forces, and

$$
\operatorname{Re}\left(\frac{\partial \Theta}{\partial t}\right)=\omega_{r} \int_{z} \theta \theta^{*} \mathrm{~d} z=\Theta_{f 1}+\Theta_{f 2}+\Theta_{d}
$$

with

$$
\Theta_{f 1}=-\operatorname{Re}\left(\int_{z} w \frac{\partial T_{0}}{\partial z} \theta^{*} \mathrm{~d} z\right)
$$

the production of fluctuating thermal energy by vertical transport of temperature,

$$
\Theta_{f 2}=-\operatorname{Re}\left(\int_{z} u \frac{\partial T_{0}}{\partial x} \theta^{*} \mathrm{~d} z\right)
$$

the production of fluctuating thermal energy by horizontal transport of temperature,

$$
\Theta_{d}=\operatorname{Re}\left(\frac{1}{\operatorname{Pr}} \int_{z} \nabla^{2} \theta \theta^{*} \mathrm{~d} z\right)
$$

the dissipation of fluctuating thermal energy by conduction.

In the above definitions, the term dissipation is used for the contributions which are by nature stabilizing (negative values), whereas the term production is used for the contributions which are by nature destabilizing (positive values) or for those which can be either stabilizing or destabilizing depending on the type of flow studied. The numerical accuracy in the calculation of the different terms of (5.1) and (5.2) is very good, as the balances expressed by (5.1) and (5.2) (respectively normalized by $\left|K_{d}\right|$ and $\left.\left|\Theta_{d}\right|\right)$ are satisfied to within $10^{-10}$. It is interesting to note that if the imaginary parts were taken in place of the real parts in the kinetic energy balance, an expression for 
the pulsation $\omega_{i}$ could be obtained. The imaginary parts associated with dissipation and magnetic terms are zero (global negative real contributions in the kinetic energy analysis). Denoting imaginary parts (associated with the real parts previously defined) by Im, we obtain

$$
\omega_{i}=\left(\operatorname{Im}\left(K_{f}\right)+\operatorname{Im}\left(K_{b}\right)\right) / \int_{z} v v^{*} \mathrm{~d} z,
$$

indicating that the pulsation $\omega_{i}$ is the sum of two terms normalized by the kinetic energy of the perturbation, one connected to the shear of the mean flow and the other to buoyancy.

The results of the energy analyses are given in the following for the two-dimensional and three-dimensional instabilities at their critical thresholds $\left(\omega_{r}=0\right)$ for thermally conducting boundary conditions. They are presented in two ways: as energy balances and as energetic contributions to the critical Grashof number.

\subsection{Energy balances}

Energy balances can be obtained from (5.1) and (5.2) for any instabilities at their critical thresholds by estimating right-hand-side terms with the corresponding critical eigenvector. Critical eigenvectors being defined to within a multiplicative constant, the terms in equations (5.1) and (5.2) are also defined to within a multiplicative constant. It is then useful to define ratios whose values will be well-defined and meaningful. Following the conventional approach, we will scale equations (5.1) and (5.2) by the dissipation terms $\left|K_{d}\right|$ and $\left|\Theta_{d}\right|$, respectively. The scaled terms are now denoted with a prime. This leads to

$$
\begin{gathered}
K_{f}^{\prime}+K_{b}^{\prime}+K_{m}^{\prime}=1, \\
\Theta_{f 1}^{\prime}+\Theta_{f 2}^{\prime}=1 .
\end{gathered}
$$

The values of these different terms are given in tables 1-4 as a function of $\mathrm{Ha}$ for the two-dimensional and three-dimensional instabilities, and for different $\mathrm{Pr}$ and different orientations of the magnetic field.

To compare the kinetic energy contributions to the thermal energy contributions, the ratio $R_{d}$ of the respective dissipation terms $K_{d}$ and $\Theta_{d}$ is also given. Comparisons will be made for a dimensionless form giving similar coefficients for the buoyancy term of (3.3) and the transport terms of (3.4) (Joseph 1976). The ratio $R_{d}$ is thus defined as

$$
R_{d}=\frac{K_{d}}{G r_{c} \Theta_{d}} .
$$

As can be checked from tables 1-4 for $H a=0$, this ratio is large (more than 1) for the two-dimensional instabilities of dynamical origin, and small (less than 1) for the three-dimensional instabilities of thermal origin.

\subsubsection{Magnetic contributions}

The magnetic contribution $K_{m}^{\prime}$ can be split into two contributions $K_{m B}^{\prime}$ and $K_{m \phi}^{\prime}$ $\left(K_{m}^{\prime}=K_{m B}^{\prime}+K_{m \phi}^{\prime}\right)$, respectively coming from the directly induced current $\boldsymbol{v} \times \boldsymbol{e}_{\boldsymbol{B} 0}$ and the potential current $-\nabla \phi$. Interesting information on these magnetic contributions can be obtained by simple considerations. The expression for the induced electric current can be written in a general form as

$$
\frac{\boldsymbol{j}}{\sigma_{e}}=\boldsymbol{E}+\boldsymbol{v} \times \boldsymbol{B} .
$$




\begin{tabular}{|c|c|c|c|c|c|c|c|}
\hline & $H a$ & $K_{f}^{\prime}$ & $K_{b}^{\prime}$ & $K_{m}^{\prime}=K_{m B}^{\prime}$ & $\Theta_{f 1}^{\prime}$ & $\Theta_{f 2}^{\prime}$ & $R_{d}$ \\
\hline \multirow[t]{10}{*}{ (a) } & 0 & 1.0012 & -0.0012 & 0 & 0.0032 & 0.9968 & 519.6 \\
\hline & 3 & 1.1106 & -0.0018 & -0.1088 & 0.0039 & 0.9961 & 352.5 \\
\hline & 5 & 1.2870 & -0.0032 & -0.2838 & 0.0052 & 0.9948 & 193.9 \\
\hline & 7 & 1.4830 & -0.0062 & -0.4768 & 0.0084 & 0.9916 & 91.56 \\
\hline & 9 & 1.5594 & -0.0120 & -0.5473 & 0.0172 & 0.9828 & 43.53 \\
\hline & 10 & 1.5361 & -0.0163 & -0.5198 & 0.0271 & 0.9729 & 31.93 \\
\hline & 11 & 1.4987 & -0.0220 & -0.4767 & 0.0442 & 0.9558 & 23.32 \\
\hline & 12 & 1.4497 & -0.0302 & -0.4195 & 0.0758 & 0.9242 & 17.06 \\
\hline & 13 & 1.4021 & -0.0424 & -0.3597 & 0.1341 & 0.8659 & 12.26 \\
\hline & 14 & 1.3656 & -0.0611 & -0.3044 & 0.2346 & 0.7654 & 8.407 \\
\hline \multirow[t]{7}{*}{ (b) } & 0 & 1.0336 & -0.0336 & 0 & 0.0816 & 0.9184 & 22.10 \\
\hline & 3 & 1.1598 & -0.0523 & -0.1075 & 0.1022 & 0.8978 & 14.34 \\
\hline & 5 & 1.3806 & -0.1049 & -0.2756 & 0.1529 & 0.8471 & 7.032 \\
\hline & 7 & 1.6816 & -0.2656 & -0.4160 & 0.3028 & 0.6972 & 2.374 \\
\hline & 9 & 1.9137 & -0.5753 & -0.3384 & 0.7007 & 0.2993 & 0.563 \\
\hline & 10 & 1.9192 & -0.6153 & -0.3040 & 0.7920 & 0.2080 & 0.256 \\
\hline & 11 & 1.9042 & -0.6396 & -0.2646 & 0.8249 & 0.1751 & 0.088 \\
\hline
\end{tabular}

TABLE 1. Energy analysis for the two-dimensional modes with vertical magnetic field at (a) $\operatorname{Pr}=0.001$ and $(b) \operatorname{Pr}=0.02\left(K_{m \phi}^{\prime}=0\right)$.

\begin{tabular}{|c|c|c|c|c|c|c|c|c|c|}
\hline & $H a$ & $K_{f}^{\prime}$ & $K_{b}^{\prime}$ & $K_{m}^{\prime}$ & $K_{m B}^{\prime}$ & $K_{m \phi}^{\prime}$ & $\Theta_{f 1}^{\prime}$ & $\Theta_{f 2}^{\prime}$ & $R_{d}$ \\
\hline \multirow[t]{10}{*}{ (a) } & 0 & 0.9520 & 0.0480 & 0 & 0 & 0 & -0.0169 & 1.0169 & 0.458 \\
\hline & 3 & 1.0694 & 0.0689 & -0.1383 & -0.2432 & 0.1049 & -0.0200 & 1.0200 & 0.374 \\
\hline & 5 & 1.2646 & 0.1144 & -0.3790 & -0.6933 & 0.3143 & -0.0256 & 1.0256 & 0.282 \\
\hline & 7 & 1.5208 & 0.1998 & -0.7206 & -1.3848 & 0.6642 & -0.0338 & 1.0338 & 0.209 \\
\hline & 9 & 1.7946 & 0.3369 & -1.1315 & -2.2874 & 1.1559 & -0.0446 & 1.0446 & 0.159 \\
\hline & 10 & 1.9251 & 0.4267 & -1.3518 & -2.7981 & 1.4463 & -0.0509 & 1.0509 & 0.141 \\
\hline & 15 & 2.4169 & 1.0731 & -2.4899 & -5.6108 & 3.1208 & -0.0899 & 1.0899 & 0.093 \\
\hline & 20 & 2.6643 & 1.9551 & -3.6195 & -8.4073 & 4.7878 & -0.1362 & 1.1362 & 0.073 \\
\hline & 25 & 2.8016 & 2.9542 & -4.7558 & -10.9512 & 6.1954 & -0.1748 & 1.1748 & 0.060 \\
\hline & 32 & 3.1024 & 4.5804 & -6.6828 & -13.9831 & 7.3004 & -0.1191 & 1.1191 & 0.033 \\
\hline \multirow[t]{7}{*}{ (b) } & 0 & 0.8206 & 0.1794 & 0 & 0 & 0 & -0.0854 & 1.0854 & 0.465 \\
\hline & 3 & 0.8974 & 0.2423 & -0.1397 & -0.2578 & 0.1181 & -0.1011 & 1.1011 & 0.400 \\
\hline & 5 & 1.0125 & 0.3642 & -0.3767 & -0.7020 & 0.3252 & -0.1269 & 1.1269 & 0.324 \\
\hline & 7 & 1.1449 & 0.5588 & -0.7038 & -1.3259 & 0.6221 & -0.1594 & 1.1594 & 0.254 \\
\hline & 9 & 1.2732 & 0.8184 & -1.0916 & -2.0639 & 0.9723 & -0.1862 & 1.1862 & 0.195 \\
\hline & 10 & 1.3357 & 0.9681 & -1.3038 & -2.4534 & 1.1496 & -0.1898 & 1.1898 & 0.167 \\
\hline & 12 & 1.4911 & 1.3228 & -1.8139 & -3.2632 & 1.4493 & -0.1342 & 1.1342 & 0.098 \\
\hline
\end{tabular}

TABLE 2. Energy analysis for the three-dimensional modes with vertical magnetic field at (a) $\operatorname{Pr}=0.02$ and (b) $\operatorname{Pr}=0.1$.

The scalar product of (5.6) with $\boldsymbol{j}$ gives after integration over the volume $\Omega$

$$
\int_{\Omega} \boldsymbol{j} \boldsymbol{E} \mathrm{d} \Omega=\int_{\Omega} \frac{\boldsymbol{j}^{2}}{\sigma_{e}} \mathrm{~d} \Omega+\int_{\Omega} \boldsymbol{v}(\boldsymbol{j} \times \boldsymbol{B}) \mathrm{d} \Omega,
$$

which can be written as

$$
P_{e}=P_{j}+P_{m}
$$




\begin{tabular}{rcccccccc}
\hline$H a$ & $K_{f}^{\prime}$ & $K_{b}^{\prime}$ & $K_{m}^{\prime}$ & $K_{m B}^{\prime}$ & $K_{m \phi}^{\prime}$ & $\Theta_{f 1}^{\prime}$ & $\Theta_{f 2}^{\prime}$ & $R_{d}$ \\
0 & 0.9520 & 0.0480 & 0 & 0 & 0 & -0.0169 & 1.0169 & 0.458 \\
3 & 0.9991 & 0.0516 & -0.0506 & -0.2351 & 0.1844 & -0.0182 & 1.0182 & 0.433 \\
7 & 1.1661 & 0.0641 & -0.2302 & -1.2840 & 1.0538 & -0.0232 & 1.0232 & 0.360 \\
10 & 1.3205 & 0.0754 & -0.3959 & -2.6229 & 2.2269 & -0.0279 & 1.0279 & 0.310 \\
20 & 1.7603 & 0.1072 & -0.8675 & -10.477 & 9.6094 & -0.0421 & 1.0421 & 0.202 \\
50 & 2.1840 & 0.1382 & -1.3222 & -65.300 & 63.977 & -0.0572 & 1.0572 & 0.092 \\
80 & 2.2597 & 0.1439 & -1.4036 & -167.08 & 165.68 & -0.0601 & 1.0601 & 0.059 \\
100 & 2.2781 & 0.1453 & -1.4234 & -261.04 & 259.61 & -0.0609 & 1.0609 & 0.047 \\
500 & 2.3104 & 0.1477 & -1.4582 & -6524.6 & 6523.2 & -0.0621 & 1.0621 & 0.010 \\
1000 & 2.3111 & 0.1478 & -1.4589 & -26099. & 26097. & -0.0622 & 1.0622 & 0.005
\end{tabular}

TABLE 3. Energy analysis for the three-dimensional modes with transverse magnetic field at $\operatorname{Pr}=0.02$.

\begin{tabular}{|c|c|c|c|c|c|c|}
\hline$H a$ & $K_{f}^{\prime}$ & $K_{b}^{\prime}$ & $K_{m}^{\prime}=K_{m B}^{\prime}$ & $\Theta_{f 1}^{\prime}$ & $\Theta_{f 2}^{\prime}$ & $R_{d}$ \\
\hline 0 & 1.0012 & -0.0012 & 0.0000 & 0.0032 & 0.9968 & 519.6 \\
\hline 3 & 1.0502 & -0.0013 & -0.0489 & 0.0034 & 0.9966 & 484.6 \\
\hline 7 & 1.1984 & -0.0016 & -0.1968 & 0.0041 & 0.9959 & 380.5 \\
\hline 10 & 1.2988 & -0.0019 & -0.2969 & 0.0045 & 0.9955 & 306.1 \\
\hline 20 & 1.4421 & -0.0024 & -0.4398 & 0.0054 & 0.9946 & 163.7 \\
\hline 50 & 1.4824 & -0.0027 & -0.4798 & 0.0057 & 0.9943 & 63.58 \\
\hline 80 & 1.4856 & -0.0027 & -0.4829 & 0.0057 & 0.9943 & 39.47 \\
\hline 100 & 1.4863 & -0.0027 & -0.4835 & 0.0057 & 0.9943 & 31.52 \\
\hline 500 & 1.4874 & -0.0028 & -0.4846 & 0.0057 & 0.9943 & 6.286 \\
\hline 1000 & 1.4874 & -0.0028 & -0.4847 & 0.0057 & 0.9943 & 3.143 \\
\hline
\end{tabular}

TABLE 4. Energy analysis for the two-dimensional modes with longitudinal magnetic field at $\operatorname{Pr}=0.001\left(K_{m \phi}^{\prime}=0\right)$.

This expression indicates that the electric power $P_{e}$ is the sum of the power dissipated by Joule effect $P_{j}$ and the power developed by the Lorentz force $P_{m}$. Replacing $\boldsymbol{E}$ by $-\nabla \phi, P_{e}$ can be expressed as

$$
P_{e}=-\int_{\Omega} \operatorname{div}(\phi \boldsymbol{j}) \mathrm{d} \Omega+\int_{\Omega} \phi \operatorname{div}(\boldsymbol{j}) \mathrm{d} \Omega,
$$

both terms of this expression being equal to zero because of the electric boundary conditions and the continuity of the electric current. The electric power $P_{e}$ is thus zero, and from (5.8), $P_{j}>0$ gives $P_{m}<0$. If we now multiply (5.6) by $\boldsymbol{E}$, we obtain after integration over the volume $\Omega$

$$
P_{e}=\int_{\Omega} \sigma_{e} \boldsymbol{E}^{2} \mathrm{~d} \Omega-\int_{\Omega} \sigma_{e} \boldsymbol{v}(\boldsymbol{E} \times \boldsymbol{B}) \mathrm{d} \Omega=0,
$$

indicating that the second term of the right-hand side, $P_{m \phi}$, is positive. As $P_{m}=$ $P_{m B}+P_{m \phi}$, we obtain that $P_{m B}$ is negative (with $\left|P_{m B}\right|>\left|P_{m}\right|$ ) which can be proved directly as this term corresponds to $\left(\sigma_{e}(\boldsymbol{v} \times \boldsymbol{B}) \times \boldsymbol{B}\right) \boldsymbol{v}=-\sigma_{e}(\boldsymbol{v} \times \boldsymbol{B})^{2}$. Finally, we can conclude that

$$
\left(K_{m}^{\prime}<0\right)=\left(K_{m B}^{\prime}<0\right)+\left(K_{m \phi}^{\prime}>0\right),
$$


i.e. the dissipation $K_{m}^{\prime}$ coming from the Lorentz force can be decomposed into a dissipative part from the directly induced current, $K_{m B}^{\prime}$, and a production part from the potential current, $K_{m \phi}^{\prime}$.

\subsubsection{Other contributions}

From tables 1-4, general considerations on the different contributions can be made.

(i) $K_{f}^{\prime}$ and $\Theta_{f 2}^{\prime}$ are positive terms corresponding to destabilizing contributions. These terms, respectively connected to the shear of the mean flow and to the horizontal transport of temperature, are in many cases dominant in their respective energy balance and so at the origin of the instabilities, $K_{f}^{\prime}$ for the two-dimensional instabilities of dynamical origin, and $\Theta_{f 2}^{\prime}$ for the three-dimensional instabilities of thermal origin.

(ii) $K_{b}^{\prime}$ and $\Theta_{f 1}^{\prime}$ have opposite signs (connected to the fact that $\partial T_{0} / \partial z$ is mainly positive), the buoyancy term $K_{b}^{\prime}$ being stabilizing for the two-dimensional instabilities due to the stable vertical stratification created by $\partial T_{0} / \partial z$, and destabilizing for the three-dimensional thermally induced instabilities.

(iii) $R_{d}$ decreases with the increase of the magnetic field intensity which indicates a more important thermal influence. This can be related to the systematic increase of $\left|K_{b}^{\prime}\right|$ and $\left|\Theta_{f 1}^{\prime}\right|$ with $H a$, and to the fact that both instabilities are limited to smaller values of $\operatorname{Pr}$ as $\mathrm{Ha}$ is increased.

\subsubsection{Energy balances for the different instabilities}

It is interesting to analyse the influence of the magnetic field on the energy balances for the different instabilities. In the following, when considering the different stabilizing and destabilizing terms of the energy balances, we will refer to their increase or decrease with regard to their absolute values.

Two-dimensional instabilities in a vertical magnetic field (table 1)

The kinetic energy balance which is dominant for two-dimensional instabilities is not strongly modified by the vertical magnetic field. There is a relatively moderate increase of the stabilizing magnetic term $K_{m}^{\prime}=K_{m B}^{\prime}$ independently of $\operatorname{Pr}$ (this term even decreasing for large enough $H a$ ), whereas the stabilizing buoyancy term $K_{b}^{\prime}$ increases too, but achieves significative values only for not too small $\mathrm{Pr}$ values $(P r=0.02)$. To compensate these increases, the destabilizing shear term $K_{f}^{\prime}$ increases too, but this increase also peaks quite quickly.

In the thermal energy balance, the main effect is the strong increase of $\Theta_{f 1}^{\prime}$, the destabilizing vertical transport of temperature, this term even becoming the dominant destabilizing term for $\mathrm{Pr}=0.02$ for large enough $\mathrm{Ha}$. This last effect seems associated with values of $R_{d}$ becoming smaller than 1 .

\section{Three-dimensional instabilities in a vertical magnetic field (table 2)}

The kinetic energy balance is strongly modified by the vertical field. Despite the destabilizing potential part $K_{m \phi}^{\prime}$, the stabilizing magnetic term $K_{m}^{\prime}$ increases regularly and strongly with $H a$, quickly becoming the main stabilizing term. To maintain the balance, the destabilizing shear and buoyancy terms $\left(K_{f}^{\prime}\right.$ and $\left.K_{b}^{\prime}\right)$ also increase strongly with $H a$. $K_{b}^{\prime}$ increases more quickly with $H a$ for larger $\operatorname{Pr}(\operatorname{Pr}=0.1)$, but the larger increase is obtained for $P r=0.02$ (calculations for large values of $H a$, in the domain where the critical curve departs from the $\mathrm{Ha}^{2}$ variation (figure 7)) where $K_{b}^{\prime}$ can become the dominant destabilizing term.

The thermal energy balance is not modified much by the vertical field. The dominant term remains the destabilizing horizontal transport of temperature $\Theta_{f 2}^{\prime}$, but, for large 


\begin{tabular}{|c|c|c|c|c|c|c|c|c|c|}
\hline & $\mathrm{Ha}$ & 0 & 3 & 7 & 10 & 20 & 50 & 80 & 100 \\
\hline (a) & $K_{m \phi}^{\prime}=-K_{m B}^{\prime}$ & 0 & 0.1647 & 0.8965 & 1.8296 & 7.3183 & 45.739 & 117.09 & 182.96 \\
\hline (b) & $K_{m \phi}^{\prime \phi}=-K_{m B}^{\prime \prime}$ & 0 & 0.0083 & 0.0450 & 0.0919 & 0.3675 & 2.2969 & 5.8800 & 9.1875 \\
\hline
\end{tabular}

TABLE 5. Magnetic contributions in the energy analysis for $(a)$ the two-dimensional modes with transverse magnetic field at $P r=0.001$, and $(b)$ the three-dimensional modes with longitudinal magnetic field at $P r=0.02$.

$\operatorname{Pr}(\operatorname{Pr}=0.1)$, the stabilizing vertical transport of temperature $\Theta_{f 1}^{\prime}$ begins to become significant with increasing $H a$.

Three-dimensional instabilities in a transverse magnetic field (table 3)

The results can be compared with those obtained in the same situation in a vertical magnetic field (table 2a). The modifications induced by the transverse magnetic field on the kinetic energy balance are less important than those by the vertical field. For the two orientations of the magnetic field, the stabilizing direct magnetic terms $K_{m B}^{\prime}$ have comparable values, but it is the more important destabilizing potential part $K_{m \phi}^{\prime}$ in the transverse case which explains the slow increase with $\mathrm{Ha}$ of the global stabilizing magnetic term $K_{m}^{\prime}$. The destabilizing terms $K_{f}^{\prime}$ and $K_{b}^{\prime}$ of the kinetic energy balance also increase more slowly in the transverse case. In fact, these modifications are necessarily limited as an asymptotic behaviour corresponding to well-defined energy balances independent of $\mathrm{Ha}$ is found for large $\mathrm{Ha}$. If the asymptotic thermal energy balance is not very different from the balance at $H a=0$ with a dominant term $\Theta_{f 2}^{\prime}$, the asymptotic kinetic energy balance is somewhat modified: there is a clear increase of $K_{m}^{\prime}$ and $K_{f}^{\prime}$, but small increase of $K_{b}^{\prime}$. Note that $K_{m}^{\prime}$ has a welldefined limit despite the fact that its components $K_{m B}^{\prime}$ and $K_{m \phi}^{\prime}$ of opposite signs both increase as $H a^{2}$. In fact, it can be observed that the magnetic term $K_{m}^{\prime}$ comprises only a small portion of $K_{m B}^{\prime}$, the potential term $K_{m \phi}^{\prime}$ more closely balancing $K_{m B}^{\prime}$ as $H a$ is increased. This will be made clearer a little later, but it can be related to the fact that the three-dimensional perturbation fields become more invariant in the transverse direction, the direction of the field $\left(k_{c}\right.$ decreases as $\mathrm{Ha}^{-1}$, and so the wavelength in this transverse direction increases as $H a$ ). Indeed, it is known that, for two-dimensional fields (with no variation in this transverse direction), $K_{m \phi}^{\prime}$ exactly balances $K_{m B}^{\prime}$. This perfect balance for two-dimensional fields also explains why there is no action of the transverse magnetic field on the two-dimensional instabilities. The energy balance in this last case (with $\mathrm{Pr}=0.001$ ) is then similar for any $\mathrm{Ha}$ to that given in table $1(a)$ for $H a=0$, except that $K_{m}^{\prime}=0$ is no longer the result of the zero values of $K_{m B}^{\prime}$ and $K_{m \phi}^{\prime}$, but of the perfect balance $K_{m \phi}^{\prime}=-K_{m B}^{\prime}$. It is interesting to see in table 5(a) that this balance occurs between very large values when $\mathrm{Ha}$ is increased (this corresponds for example for $H a=100$ to values of $K_{m B}$ almost 183 times the values of the dissipation term $K_{d}$ ). Such large values explain why more realistic situations with even a small departure from two-dimensionality are affected by non-negligible stabilizing magnetic effects.

It has been verified from the variation of the critical eigenvectors that the asymptotic energy balances for large $\mathrm{Ha}$ corresponded to clear asymptotic variations at the level of the flow characteristics, namely $u / w \sim \theta / w \sim v / w \sim \phi / w \sim H a$. The ratio between $v$ and $w$ is directly connected to flow conservation in the yoz-plane, having in mind that the length of the cell (along $y$ ) evolves as $H a$. 
A small order of magnitude analysis can explain the details of the magnetic contributions to the energy analysis. For the three-dimensional instabilities in a transverse magnetic field, the Lorentz force is equal to $H a^{2}\left((-u+\partial \phi / \partial z) \boldsymbol{e}_{x}-w \boldsymbol{e}_{z}\right)$, so that there are two contributions for $K_{m B}^{\prime}$ and one for $K_{m \phi}^{\prime}$. From the asymptotic variations of the flow characteristics, we find that the contribution of $K_{m B}^{\prime}$ coming from $-\mathrm{Ha}^{2} w$ scales as $\mathrm{Ha}^{2} \mathrm{O}\left(\mathrm{Ha}^{-2}\right) \sim O(1)$ and is asymptotically equal to -0.0708 , a small part of $K_{m}^{\prime}$, whereas the two other contributions scale as $H a^{2} O(1) \sim O\left(H a^{2}\right)$. But, from the conservation of current, $-k^{2} \phi+\partial^{2} \phi / \partial z^{2}=\partial u / \partial z$, we can deduce that $-u+\partial \phi / \partial z \sim H a^{-2} \phi$, so that the two other contributions together scale as $\mathrm{Ha}^{2} \mathrm{O}\left(\mathrm{Ha}^{-2}\right) \sim \mathrm{O}(1)$. The corresponding asymptotic value is 1.388 and constitutes the main part of $K_{m}^{\prime}$. Despite the fact that the quantity $-u+\partial \phi / \partial z$ goes to zero with the lengthening of the marginal cells, the asymptotic value obtained is not zero because this quantity only goes to zero as $\mathrm{Ha}^{-2}$.

Finally, in this case, we have used expression (5.3) to calculate contributions to $\omega_{i}$, without a magnetic field $(\mathrm{Ha}=0)$ and in the asymptotic domain for large $\mathrm{Ha}$ $(H a=1000)$. For $H a=0, \omega_{i}=261.09$ with 252.12 coming from the shear term and 8.97 from the buoyancy term, whereas for $H a=1000, \omega_{i}=413.74$ with 397.42 and 16.32 for the respective contributions. The main contribution always comes from the shear term and both contributions have increased with the increase of $\mathrm{Ha}$.

\section{Two-dimensional instabilities in a longitudinal magnetic field (table 4)}

The energy analysis shows that $K_{b}^{\prime}$ and $\Theta_{f 1}^{\prime}$ remain small and so the dissipation terms are equilibrated by $K_{f}^{\prime}$ and $K_{m}^{\prime}$ in the kinetic energy balance and by $\Theta_{f 2}^{\prime}$ in the thermal energy balance. Compared to the case of the vertical magnetic field (table $1 a$ ), the initial evolution with $H a$ of $K_{f}^{\prime}$ and $K_{m}^{\prime}$ is less pronounced. For large $H a$, as for the three-dimensional modes with transverse magnetic field, asymptotic energy balances are found which are not very different from the balance at $H a=0$ (moderate increase of $K_{m}^{\prime}$ ).

The perfect balance between $K_{m \phi}^{\prime}$ and $K_{m B}^{\prime}$ explains why there is no action of the longitudinal magnetic field on the three-dimensional instabilities. The values $K_{m \phi}^{\prime}=-K_{m B}^{\prime}$ given in table $5(b)$ become important for large $H a$ but less than for the two-dimensional instabilities in a transverse magnetic field.

Finally, in this case also, the asymptotic energy balances for large $\mathrm{Ha}$ correspond to clear asymptotic variations at the level of the flow characteristics, namely $u / w \sim$ $\theta / w \sim H a$.

\subsection{Energetic contributions to the critical Grashof number}

The previous analysis has shown how the energy balances between the different production and dissipation terms evolve when a magnetic field is applied. It would also be interesting to understand what are the main reasons for the very different stabilizing effect obtained in the different cases studied, particularly the relative importance of on the one hand the modification of the basic flow by the magnetic field and on the other hand the Lorentz term. For that, it is convenient to present the energy analysis results in a slightly different way. We know that the basic flow $U_{0}$ is proportional to $G r: U_{0}=G r v_{0}$, where $v_{0}=v_{0}(z)$ is a normalized profile taking into account the modifications due to the magnetic field. We then define $k_{f}$ based on $v_{0}$ such that $K_{f}=G r k_{f}$ and $k_{b}$ such that $K_{b}=G r k_{b}$, and so after division by $\left|K_{d}\right|$, we can express (5.1) at the critical threshold as

$$
G r_{c}\left(k_{f}^{\prime}+k_{b}^{\prime}\right)=1-K_{m}^{\prime} .
$$




\begin{tabular}{rrrrrrrr}
\hline & \multicolumn{3}{c}{$\operatorname{Pr}=0.001$} & & \multicolumn{3}{c}{$\operatorname{Pr}=0.02$} \\
\cline { 2 - 3 }$H a$ & $R_{1}$ & $R_{2}$ & $G r_{c} / G r_{c_{0}}$ & & $R_{1}$ & $R_{2}$ & $G r_{c} / G r_{c_{0}}$ \\
3 & 1.36 & 1.11 & 1.51 & & 1.39 & 1.11 & 1.54 \\
5 & 2.25 & 1.28 & 2.88 & & 2.46 & 1.28 & 3.14 \\
7 & 4.81 & 1.48 & 7.10 & & 7.07 & 1.42 & 10.01 \\
9 & 14.79 & 1.55 & 22.88 & & 39.22 & 1.34 & 52.49 \\
10 & 28.47 & 1.52 & 43.27 & & 88.50 & 1.30 & 115.40 \\
11 & 56.54 & 1.48 & 83.48 & 237.58 & 1.26 & 300.44 \\
12 & 115.89 & 1.42 & 164.51 & & & \\
13 & 239.76 & 1.36 & 325.99 & & & \\
14 & 491.09 & 1.30 & 640.60 & & &
\end{tabular}

TABLE 6. Characterization of the stabilization by a vertical magnetic field for the two-dimensional modes at different $\mathrm{Pr}$.

As $K_{m}^{\prime}$ is negative, the action of the magnetic field will first increase $G r_{c}$ through the increase of the right-hand side in connection with the Lorentz force. It will also modify the $v_{0}$ profile which will generate less shear, leading to a decrease of $k_{f}^{\prime}$. This time, $G r_{c}$ will increase in order to maintain the equality (5.11). In order to have a better estimation of the action of the magnetic field, we will compare $G r_{c}$ to $G r_{c_{0}}$, the critical value obtained for $H a=0$. We can write

$$
G r_{c_{0}}\left(\frac{k_{f_{0}}+k_{b_{0}}}{\left|K_{d_{0}}\right|}\right)=1
$$

where the values with the subscript 0 refer to the case $H a=0$, so that (5.11) can also be expressed as

$$
\frac{G r_{c}}{G r_{c_{0}}}=R_{1} R_{2}
$$

where

$$
R_{1}=\left(\frac{k_{f_{0}}^{\prime}+k_{b_{0}}^{\prime}}{k_{f}^{\prime}+k_{b}^{\prime}}\right), \quad R_{2}=1-K_{m}^{\prime} .
$$

$R_{1}$ and $R_{2}$ are always larger than 1 (being equal to 1 for $H a=0$ ), $R_{1}$ referring to the increase mainly due to the modification of the $v_{0}$ profile (the buoyancy term $k_{b}^{\prime}$ is often negligible compared to $k_{f}^{\prime}$, except for the three-dimensional instabilities in a vertical magnetic field at large $H a$, see $K_{b}^{\prime}$ and $K_{f}^{\prime}$ in tables $\left.1-4\right)$, and $R_{2}$ referring to the increase due to the Lorentz force $\left(R_{2}\right.$ is directly connected to $K_{m}^{\prime}$ given in the previous tables). The results are presented in tables 6-8 where for each case the evolutions with $\mathrm{Ha}$ of $R_{1}, R_{2}$ and $G r_{c} / G r_{c_{0}}=R_{1} R_{2}$ are given.

\subsubsection{Two- and three-dimensional instabilities under vertical magnetic field (tables 6 and 7)}

The results clearly show the differences between the stabilization of the twodimensional and three-dimensional modes in a vertical magnetic field. For the twodimensional modes (table 6), $R_{2}$ remains rather small (the maximum values reached are close to 1.55 , the value at $H a=9$, for $P r=0.001$ and close to 1.42 , the value at $H a=7$, for $P r=0.02$ ) whereas $R_{1}$ increases strongly and regularly with $H a$, reaching for example for $H a=10$ the values 28.47 for $\mathrm{Pr}=0.001$ and 88.5 for $\mathrm{Pr}=0.02$. For the three-dimensional modes (table 7), $R_{2}$ and $R_{1}$ both increase regularly with $\mathrm{Ha}$ but remain moderate, except for the largest $H a$. Compared to the two-dimensional 


\begin{tabular}{rccccccc}
\hline & \multicolumn{3}{c}{$P r=0.02$} & & \multicolumn{3}{c}{$\operatorname{Pr}=0.1$} \\
\cline { 2 - 4 } \cline { 6 - 7 } & $R_{1}$ & $R_{2}$ & $G r_{c} / G r_{c_{0}}$ & & $R_{1}$ & $R_{2}$ & $G r_{c} / G r_{c_{0}}$ \\
3 & 1.07 & 1.14 & 1.22 & & 1.11 & 1.14 & 1.27 \\
5 & 1.16 & 1.38 & 1.60 & 1.30 & 1.38 & 1.79 \\
7 & 1.28 & 1.72 & 2.20 & 1.60 & 1.70 & 2.73 \\
9 & 1.42 & 2.13 & 3.02 & 2.08 & 2.09 & 4.35 \\
10 & 1.50 & 2.35 & 3.52 & 2.44 & 2.30 & 5.62 \\
12 & 1.68 & 2.80 & 4.72 & 3.98 & 2.81 & 11.19 \\
15 & 2.04 & 3.49 & 7.14 & & & \\
20 & 2.89 & 4.62 & 13.34 & & & \\
25 & 4.19 & 5.76 & 24.11 & & & \\
32 & 8.81 & 7.68 & 67.67 & & &
\end{tabular}

TABLE 7. Characterization of the stabilization by a vertical magnetic field for the three-dimensional modes at different $P r$.

\begin{tabular}{rrrrrrrr}
\hline & \multicolumn{3}{c}{$(a)$} & & \multicolumn{3}{c}{$(b)$} \\
\cline { 2 - 3 }$H a$ & $R_{1}$ & $R_{2}$ & $G r_{c} / G r_{c_{0}}$ & & $R_{1}$ & $R_{2}$ & $G r_{c} / G r_{c_{0}}$ \\
3 & 1.00 & 1.05 & 1.05 & & 1.01 & 1.05 & 1.06 \\
7 & 1.00 & 1.23 & 1.23 & & 1.09 & 1.20 & 1.31 \\
10 & 1.01 & 1.40 & 1.41 & & 1.21 & 1.30 & 1.58 \\
20 & 1.16 & 1.87 & 2.17 & & 1.87 & 1.44 & 2.69 \\
50 & 2.08 & 2.32 & 4.83 & & 4.35 & 1.48 & 6.44 \\
80 & 3.16 & 2.40 & 7.61 & & 6.91 & 1.48 & 10.25 \\
100 & 3.91 & 2.42 & 9.47 & & 8.63 & 1.48 & 12.80 \\
500 & 19.14 & 2.46 & 47.06 & & 43.05 & 1.48 & 63.91 \\
1000 & 38.27 & 2.46 & 94.09 & 86.08 & 1.48 & 127.81
\end{tabular}

TABLE 8. Characterization of the stabilization by $(a)$ a transverse magnetic field for the three-dimensional modes at $\operatorname{Pr}=0.02$, and $(b)$ a longitudinal magnetic field for the twodimensional modes at $\mathrm{Pr}=0.001$.

modes, $R_{2}$ is somewhat larger, reaching values around 2.3 for $H a=10$, whereas $R_{1}$ is much smaller, reaching the values 1.5 and 2.44 at $H a=10$, for respectively $P r=0.02$ and $\operatorname{Pr}=0.1$. All this indicates that the very good stabilization obtained for the twodimensional modes is mainly the consequence of the strong diminution of the energy generated by shear when the velocity profiles are modified by a vertical magnetic field (values of $R_{1}$ up to 491 have been calculated at $P r=0.001$ for values of $H a$ as small as 14), and only slightly connected to the stabilizing effect of the Lorentz force which remains very weak. The stabilization is weaker for the three-dimensional modes as the effect of shear remains moderate (the increase of $R_{1}$ is even in part connected to the increase of $K_{b}^{\prime}$ ), despite the fact that the Lorentz force effect is stronger. The increase of $\operatorname{Pr}$ is found to increase the stabilizing effect of the magnetic field, mainly through the term $R_{1}$.

The strong stabilization of the two-dimensional modes has been connected to the modifications induced by the vertical magnetic field on the velocity profiles. In fact, as shown in figure 2 , for the range of $H a$ considered $(H a \leqslant 30)$, the global characteristics such as the intensity of the flow and the position of the maximum velocity do not change very quickly with $\mathrm{Ha}$ (the intensity is roughly divided by 4 for $\mathrm{Ha}=10$ 


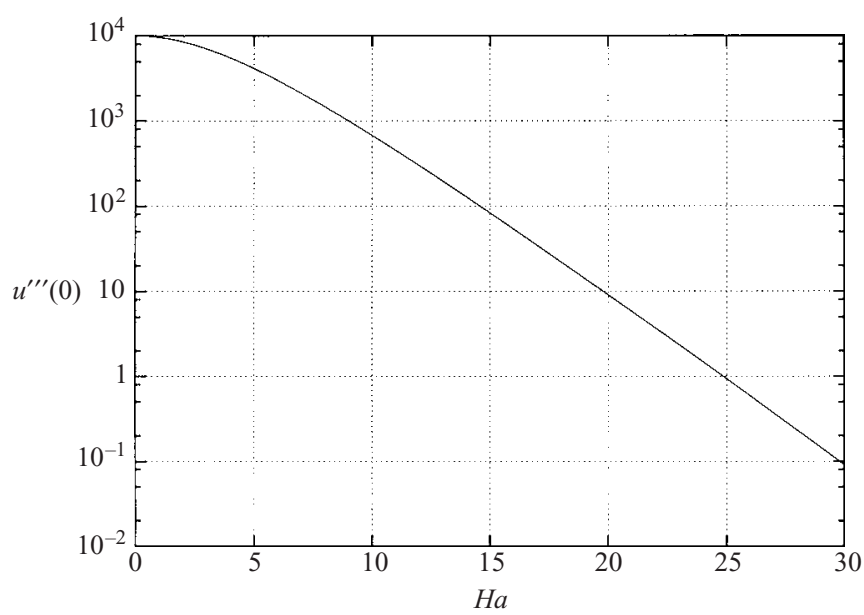

FIGURE 16. Variation of the third derivative of the basic velocity profiles at the inflection point $\left(u^{\prime \prime \prime}(0)=\partial^{3} u / \partial z^{3}(0)\right)$ as a function of $H a$.

and by 20 for $\mathrm{Ha}=30$, and the position evolves slowly). Having in mind that the simple criteria for the instability of parallel two-dimensional flows in the framework of the inviscid theory are connected to the existence of an inflection point in the velocity profile (Rayleigh's and Fjortoft's theorems, see Drazin \& Reid 1981), it could be interesting to look at the second derivative $u^{\prime \prime}=\partial^{2} u / \partial z^{2}$ in our profiles. Our profiles are symmetric, always with an inflection point in the middle at $z=0$ and a second derivative varying from 0 at $z=0$ to 5000 at the upper boundary at $z=0.5$. The key factor for the instability could be the variation of the second derivative around the inflection point (estimated through the third derivative at the inflection point, $\left.u^{\prime \prime \prime}(0)=\partial^{3} u / \partial z^{3}(0)\right)$ as this quantity has been found to vary very fast with $\mathrm{Ha}$ : figure 16 shows that it decreases by 5 decades for $\mathrm{Ha}$ only changing from 0 to 30 , with a clear asymptotic variation beyond $H a=20$ as $u^{\prime \prime \prime}(0) \sim \exp (-0.46 \mathrm{Ha})$.

\subsubsection{Three-dimensional instabilities in a transverse magnetic field (table 8a) and two-dimensional instabilities in a longitudinal magnetic field (table 8b)}

In these cases where the basic velocity (and temperature) profiles are not modified by the magnetic field (inducing a very small initial increase of $R_{1}$ ), the stabilizations obtained are less effective than with a vertical field, but they grow to large $\mathrm{Ha}$ with an asymptotic variation in $\mathrm{Ha}$.

For the three-dimensional modes in a transverse magnetic field (table $8 a$ ), $R_{1}$ remains close to 1 until $H a=10$, the observed stabilization only coming from the Lorentz term. For larger values of $\mathrm{Ha}$, despite the invariance of the basic flow, $R_{1}$ begins to increase, reaching an asymptotic $H a$ variation for large values of $H a$, whereas the Lorentz term levels off above $H a=50$ and reaches an asymptotic value.

For the two-dimensional modes in a longitudinal magnetic field (table $8 b$ ), the stabilization is somewhat larger than for the transverse field. $R_{1}$ evolves more quickly for small $\mathrm{Ha}$ before reaching the asymptotic $\mathrm{Ha}$ variation for large $\mathrm{Ha}$, whereas $R_{2}$ evolves more slowly and reaches a smaller asymptotic value.

These asymptotic variations of $\mathrm{Gr}_{c}$ at large $\mathrm{Ha}$ can be found through an order of magnitude analysis of the perturbation equations. We will consider the simplest case, the two-dimensional modes in a longitudinal magnetic field, and choose the limit $\operatorname{Pr}=0$. In that case, we have $\partial \boldsymbol{v} / \partial t=0, v=0$ and $\theta=0$. Equation (3.3) can then be 
written as

$$
\left(\boldsymbol{V}_{0} \cdot \nabla\right) \boldsymbol{v}+(\boldsymbol{v} \cdot \nabla) \boldsymbol{V}_{0}=-\nabla p+\nabla^{2} \boldsymbol{v}+H a^{2} \boldsymbol{j} \times \boldsymbol{e}_{\boldsymbol{B} 0},
$$

with

$$
\boldsymbol{j}=-\nabla \phi+\boldsymbol{v} \times \boldsymbol{e}_{\boldsymbol{B} 0} .
$$

Similarly to Alboussière et al. $(1993,1997)$, the curl operator has to be applied twice to $(5.15)$ and once to (5.16). Direct substitution then leads to

$$
\Delta^{2} \boldsymbol{v}+H a^{2}\left(\boldsymbol{e}_{\boldsymbol{B} 0} \cdot \nabla\right)^{2} \boldsymbol{v}=\left(\nabla \times\left(\nabla \times\left(\left(\boldsymbol{V}_{0} \cdot \nabla\right) \boldsymbol{v}+(\boldsymbol{v} \cdot \nabla) \boldsymbol{V}_{0}\right)\right)\right),
$$

where $\Delta$ is the Laplacian operator (here, $\Delta=\partial^{2} / \partial z^{2}-h^{2}$ ). Knowing that for the two-dimensional transverse instabilities $\left(\boldsymbol{V}_{0} \cdot \nabla\right) \boldsymbol{v}=U_{0} \mathrm{i} h u \boldsymbol{e}_{x}+U_{0} \mathrm{i} h w \boldsymbol{e}_{z}$ and $(\boldsymbol{v} \cdot \nabla) \boldsymbol{V}_{0}=w\left(\partial U_{0} / \partial z\right) \boldsymbol{e}_{x}$, the right-hand side of (5.17) can be calculated. We will consider the real part of equation (5.17) projected on $\boldsymbol{e}_{z}$. With $\boldsymbol{e}_{\boldsymbol{B} 0}=\boldsymbol{e}_{x}$ (longitudinal field), we obtain

or

$$
\Delta^{2} w+H a^{2} \frac{\partial^{2} w}{\partial x^{2}}=-h^{2} \frac{\partial}{\partial z}\left(U_{0} u\right),
$$

$$
\Delta^{2} w=h^{2}\left(H a^{2} w-\frac{\partial}{\partial z}\left(U_{0} u\right)\right) .
$$

We assume now that $H a$ is large $(H a \gg 1)$, that the length of the marginal cell is much larger than the depth of the layer $(2 \pi / h \gg 1$ in a dimensionless form, so that $\left.\Delta \sim \partial^{2} / \partial z^{2} \sim O(1)\right)$, and that $\left(\partial\left(U_{0} u\right) / \partial z\right) / w \sim O\left(H a^{2}\right)$ (this will be verified in the following). Equation (5.19) then leads to a scaling for $h$, namely

$$
h \sim H a^{-1}
$$

and flow conservation for the perturbation in the $x o z$-plane gives

$$
\frac{u}{w} \sim H a
$$

Using (5.11), it is now possible to obtain a scaling for $G r_{c}$. For the two-dimensional instabilities in a longitudinal magnetic field, the Lorentz force is reduced to $-H a^{2} w \boldsymbol{e}_{z}$, so that

$$
K_{m}=H a^{2} \operatorname{Re}\left(\int_{z}-w w^{*} \mathrm{~d} z\right) .
$$

Knowing that

$$
K_{d} \sim \operatorname{Re}\left(\int_{z} \frac{\partial^{2} u}{\partial z^{2}} u^{*} \mathrm{~d} z\right)
$$

and

$$
k_{f}=-\operatorname{Re}\left(\int_{z} w \frac{\partial v_{0}}{\partial z} u^{*} \mathrm{~d} z\right),
$$

and using relation (5.21) and the fact that $\partial v_{0} / \partial z \sim O(1), K_{m}^{\prime}$ and $k_{f}^{\prime}$ can be estimated. We obtain

$$
K_{m}^{\prime}=\frac{K_{m}}{K_{d}} \sim O(1)
$$

and

$$
k_{f}^{\prime}=\frac{k_{f}}{K_{d}} \sim H a^{-1} .
$$


Using the fact that $k_{b}^{\prime}=0$ for $\operatorname{Pr}=0$, equation (5.11) leads to the scaling for $G r_{c}$

$$
G r_{c} \sim H a .
$$

It can be checked that the hypothesis $\left(\partial\left(U_{0} u\right) / \partial z\right) / w \sim O\left(H a^{2}\right)$ is verified well as it is found that $\left(\partial\left(U_{0} u\right) / \partial z\right) / w \sim G r u / w \sim H a^{2}$. From this analysis, it is shown that the asymptotic variation of the threshold for the two-dimensional instabilities in a longitudinal magnetic field is driven by the increase of the length of the marginal cells. The key factor is the decrease of the main destabilizing shear energy term $k_{f}^{\prime}$ connected here to the decrease of the vertical component of the velocity perturbation compared to the horizontal component, and not to the mean flow which is unchanged. A similar but more complex analysis could be done for the three-dimensional instabilities in a transverse magnetic field.

\section{Concluding remarks}

We have characterized the stabilization of buoyant parallel flows (flows generated between infinite horizontal walls by a horizontal temperature gradient) by a uniform magnetic field (vertical, or horizontal with a direction transverse or longitudinal to the flow) through a linear stability analysis and energy considerations. The main results can be summarized as follows:

(i) The vertical magnetic field (for which the basic flow profiles are modified by the field) stabilizes the instabilities more quickly than the horizontal fields, but the stabilization is only obtained up to moderate values of $\mathrm{Ha}$ (before the disappearance of the instabilities) whereas for the horizontal fields the stabilization is effective up to large values of $\mathrm{Ha}$.

(ii) The vertical magnetic field strongly stabilizes the two-dimensional instabilities of dynamic origin, giving initial variations of the critical Grashof number $G r_{c}$ at small $H a$ as $G r_{c_{0}} \exp \left(H a^{2}\right)$. This is due to the strong decrease of the main destabilizing term $k_{f}^{\prime}$ (connected to fluctuating energy generated by shear of the mean flow) when the velocity profiles are modified by the vertical magnetic field. On the other hand, the stabilizing effect of the Lorentz force remains very weak. The wavenumber $h_{c}$ associated with these instabilities decreases with $\mathrm{Ha}$. The kinetic energy balance is only slightly modified, whereas the thermal energy balance clearly changes.

(iii) The vertical magnetic field stabilizes the three-dimensional instabilities of thermal origin less quickly than the two-dimensional instabilities, giving initial variations of $G r_{c}$ at small $H a$ as $G r_{c}-G r_{c_{0}} \sim H a^{2}$. As a result, as $H a$ increases, the range of Prandtl numbers over which the three-dimensional oscillatory instabilities are the preferred modes extends progressively towards lower values of the Prandtl number. For these three-dimensional instabilities, the effect of shear remains moderate, but the Lorentz force effect is stronger. The wavenumber $k_{c}$ is found to increase with $H a$, the opposite of the case of two-dimensional instabilities. The kinetic energy balance is strongly modified, whereas the thermal energy balance is only slightly changed.

(iv) Asymptotic behaviour of $G r_{c}$ as $G r_{c} \sim H a$ is found for the horizontal fields. These asymptotic increases are due to the decrease of the main destabilizing shear energy term $k_{f}^{\prime}$ and connected to the asymptotic increase of the marginal cell length (decrease of the wavenumbers $h_{c}$ or $k_{c}$ as $\mathrm{Ha}^{-1}$ ). The energy balances evolve towards well-defined asymptotic balances, with more important changes for the kinetic energy balances.

(v) Somewhat larger stabilization is found for the two-dimensional modes in a longitudinal field than for the three-dimensional modes in a transverse field, connected 
to a stronger decrease of $k_{f}^{\prime}$. The stabilizing action of the Lorentz term is stronger for the three-dimensional modes, despite the fact that destabilizing potential effects are present in this case.

Comparisons can be made between this infinite-layer model and more realistic finite-length situations. First, concerning the infinite-layer parallel flow solution, it has been suggested that in a magnetic field this solution may be representative of the longitudinal main flow only at moderate $\mathrm{Ha}$, principally for the vertical field, but also for the longitudinal field if the cavity is quite long in this longitudinal direction. Then, concerning the stabilizing effect of the magnetic field, numerical results in a twodimensional rectangular cavity (Gelfgat \& Bar-Yoseph 2001) as well as experimental results in a parallelepipedic cavity (Hof 2001) have shown a very strong increase of the instability thresholds for all directions of the magnetic field. In Hof (2001), the increase is explicitly given as exponential variations, whereas in Gelfgat \& BarYoseph (2001) the increase (first increase from $H a=0$ ) is not characterized by the authors but also looks exponential. In both studies, the instabilities are connected to shear effects in the longitudinal main flow, and this flow is modified by the magnetic field, whatever its direction. This is similar to what occurs in the layer for the twodimensional instabilities in a vertical magnetic field, a situation where an exponential stabilizing effect has also been found.

The authors would like to express their grateful thanks to Dr T. Alboussière from the University of Cambridge, Professor R. Moreau from the MADYLAM of Grenoble, and Dr J. P. Garandet from CENG for fruitful discussions. We also wish to thank Dr P. Laure from the INL Nice for helpful suggestions about the numerical calculations and Professor R. L. Sani from the University of Colorado for valuable advice. This work was supported by the CNES through the microgravity program.

\section{REFERENCES}

Alboussière, T., Garandet, J. P. \& Moreau, R. 1993 Buoyancy-driven convection with a uniform magnetic field. Part 1. Asymptotic analysis. J. Fluid Mech. 253, 545.

Alboussière, T., Neubrand, A. C., Garandet, J. P. \& Moreau, R. 1997 Segregation during horizontal Bridgman growth under an axial magnetic field. J. Cryst. Growth 181, 133.

Aleksandrova, S. \& Molokov, S. 2000 Three-dimensional buoyant convection in a rectangular cavity with differentially headed walls in a strong magnetic field. Proc. Third Intl Symp. on Electromagnetic Processing of Materials, Nagoya, Japan, pp. 153-158. ISIJ Publications.

BenHadid, H. \& Roux, B. 1992 Buoyancy- and thermocapillary-driven flows in differentially heated cavities for low-Prandtl-number fluids. J. Fluid Mech. 235, 1.

BenHadid, H. \& Henry, D. 1994 Numerical simulation of convective three-dimensional flows in a horizontal Bridgman configuration under the action of a constant magnetic field. Proc. Second Intl Conf. on Energy Transfer in MagnetoHydroDynamic Flows, Aussois, France, Vol. 1, p. 47. MHD Pamir Publications.

BenHadid, H. \& HenRy, D. 1996 Numerical simulation of convective three-dimensional flows in a horizontal cylinder under the action of a constant magnetic field. J. Cryst. Growth 166, 436.

BenHadid, H. \& Henry, D. 1997 Numerical study of convection in the horizontal Bridgman configuration under the action of a constant magnetic field. Part 2. Three-dimensional flows. J. Fluid Mech. 333, 57.

BenHadid, H., Henry, D. \& Kaddeche, S. 1997 Numerical study of convection in the horizontal Bridgman configuration under the action of a constant magnetic field. Part 1. Two-dimensional flows. J. Fluid Mech. 333, 23. 
Bessaih, R., Kadja, M. \& Marty, Ph. 1999 Effect of wall electrical conductivity and magnetic field orientation on liquid metal flow in a geometry similar to the horizontal Bridgman configuration for crystal growth. Intl J. Heat Mass Transfer 42, 4345.

BoJAREVICS, V. 1995 Horizontal temperature gradient driven flow and its stability in a horizontal channel of rectangular cross-section with the effects of transversal magnetic field. In Advances in Engineering Heat Transfer, Proc. Second Baltic Heat Transfer Conf. (ed. B. Sunden, E. Blums, A. Zukauskas). Computational Mechanics Publications, Southampton.

ChandraseKhar, S. 1961 Hydrodynamic and Hydromagnetic Stability. Clarendon.

Cormack, D. E., Leal, L. G. \& Imberger, J. 1974 Natural convection in a shallow cavity with differentially heated end walls. Part 1. Asymptotic theory J. Fluid Mech. 65, 209.

Davoust, L., Cowley, M. D., Moreau, R. \& Bolcato, R. 1999 Buoyancy driven convection with a uniform magnetic field. Part 2. Experimental investigation. J. Fluid Mech. 400, 59.

Di Piazza, I. \& Ciofalo, M. 2002 MHD free convection in a liquid-metal filled cubic enclosure. I. Differential heating. Intl J. Heat Mass Transfer 45, 1477.

Drazin, P. G. \& ReID, W. H. 1981 Hydrodynamic Stability. Cambridge University Press.

Gelfgat, A. Yu. \& BAR-YosePh, P.Z. 2001 The effect of an external magnetic field on oscillatory instability of convective flows in a rectangular cavity. Phys. Fluids 13, 2269.

Gershuni, G. Z. \& Zhukhovitsky, E. M. 1976 Convective Stability of Incompressible Fluids. Israel Program for Scientific Translations, Keter Publishing House, Jerusalem.

Gershuni, G.Z., Laure, P., Myznikov, V.M., Roux, B. \& Zhukhovitsky, E. M. 1992 On the stability of plane-parallel advective flows in long horizontal layers. Microgravity Q. 2, 141.

GiLl, A. E. 1974 A theory of thermal oscillations in liquid metals. J. Fluid Mech. 64, 577.

Hart, J.E. 1972 Stability of thin non-rotating Hadley circulations. J. Atmos. Sci. 29, 687.

Hart, J.E. 1983 A note on stability of low Prandtl number Hadley circulation. J. Fluid Mech. 132, 271.

HoF, B. 2001 A study of magnetohydrodynamic convection in liquid gallium. Thesis, University of Manchester.

Hung, M. C. \& ANDERECK, C. D. 1988 Transitions in convection driven by a horizontal temperature gradient. Phys. Lett. A 132, 253.

Hurle, D. T. J. 1966 Temperature oscillations in molten metals and their relationship to growth striae in melt-grown crystals. Phil. Mag. 13(122), 305.

Hurle, D. T. J., JaKeman, E. \& Johnson, C.P. 1974 Convective temperature oscillations in molten gallium. J. Fluid Mech. 64, 565.

JosePH, D. D. 1976 Stability of Fluid Motions II. Springer.

Juel, A., Mullin, T., BenHadid, H. \& Henry, D. 1999 Magnetohydrodynamic convection in molten gallium. J. Fluid Mech. 378, 97.

Kim, D. H., Adornato, P. \& Brown, R. 1988 Effect of vertical magnetic field on convection and segregation in vertical Bridgman crystal growth. J. Cryst. Growth 89, 339.

Laure, P. \& Roux, B. 1987 Synthèse des résultats obtenus par l'étude de stabilité des mouvements de convection dans une cavité horizontale de grande extension. C. R. Acad. Sci. Paris 305, 1137.

LAure, P. \& Roux, B. 1989 Linear and non-linear analysis of Hadley circulation. J. Cryst. Growth 97, 226.

Oreper, G. M. \& Szekely, J. 1983 The effect of an externally imposed magnetic field on buoyancy driven flow in a rectangular cavity. J. Cryst. Growth 64, 505.

OrePer, G. M. \& Szekely, J. 1984 The effect of a magnetic field on transport phenomena in a Bridgman-Stockbarger crystal growth. J. Cryst. Growth 67, 405.

OzoE, H. \& OKada, K. 1989 The effect of the direction of the external magnetic field on the three-dimensional natural convection in a cubical enclosure. Intl J. Heat Mass Transfer 32, 1939.

Parmentier, P. M., RÉGnier, V.C. \& Lebon, G. 1993 Buoyant-thermocapillary instabilities in medium-Prandtl-number fluid layers subjected to horizontal temperature gradient. Intl $J$. Heat Mass Transfer 36, 2417.

PimputKar, S. M. \& Ostrach, S. 1981 Convective effects in crystals grown from melts. J. Cryst. Growth 55, 614.

Priede, J. \& Gerbeth, G. 1995 Hydrothermal wave instability of thermocapillary driven convection in a plane layer subjected to a uniform magnetic field. Adv. Space Res. 16, 55. 
Priede, J. \& Gerbeth, G. $1997 a$ Hydrothermal wave instability of thermocapillary-driven convection in a coplanar magnetic field. J. Fluid Mech. 347, 141.

Priede, J. \& Gerbeth, G. 1997b Influence of thermal boundary conditions on the stability of thermocapillary-driven convection at low Prandtl numbers. Phys. Fluids 9, 1621.

Priede, J. \& Gerbeth, G. 2000 Hydrothermal wave instability of thermocapillary-driven convection in a transverse magnetic field. J. Fluid Mech. 404, 211.

Priede, J., Thess, A. \& Gerbeth, G. 1994 Thermocapillary instabilities in liquid metals: Hartmann-number versus Prandtl-number. Proc. Second Intl Conf. on Energy Transfer in MagnetoHydroDynamic Flows, Aussois, France, Vol. 2, p. 571. MHD Pamir Publications.

Smith, M. K. \& Davis, S.H. 1983 Instabilities of dynamic thermocapillary liquid layers. Part I: Convective instabilities $J$. Fluid Mech. 132, 119.

Tagawa, T. \& OzoE, H. 1998a Enhanced heat transfer rate measured for natural convection in liquid gallium in a cubical enclosure under a static magnetic field. Trans. ASME: J. Heat Transfer 120, 1027.

TAGaWA, T. \& OzoE, H. $1998 \mathrm{~b}$ The natural convection of liquid metal in a cubical enclosure with various electro-conductivities of the wall under the magnetic field. Intl J. Heat Mass Transfer 41, 1917. 